\title{
Nonempirical dielectric-dependent hybrid functional with range separation for semiconductors and insulators
}

\author{
Wei Chen, ${ }^{1,2}$ Giacomo Miceli, ${ }^{1}$ Gian-Marco Rignanese, ${ }^{2}$ and Alfredo Pasquarello ${ }^{1}$ \\ ${ }^{1}$ Chaire de Simulation à l'Echelle Atomique (CSEA), Ecole Polytechnique Fédérale de Lausanne (EPFL), CH-1015 Lausanne, Switzerland \\ ${ }^{2}$ Institute of Condensed Matter and Nanoscicence (IMCN), Université catholique de Louvain, B-1348 Louvain-la-Neuve, Belgium
}

(Received 4 November 2017; revised manuscript received 4 June 2018; published 24 July 2018)

\begin{abstract}
We present a general scheme of range-separated hybrid functionals in which the mixing parameters of Fock exchange are fully nonempirical and determined solely from the dielectric function. We show that the full dielectric dependence leads to an unscreened Fock exchange in the short range, while in the long range the Fock exchange is correctly screened by the macroscopic dielectric constant. The range separation is obtained by fitting to the calculated static dielectric function in the long-wavelength limit. The resulting hybrid functional accurately accounts for electronic and structural properties of various semiconductors and insulators spanning a wide range of band gaps.
\end{abstract}

DOI: 10.1103/PhysRevMaterials.2.073803

\section{INTRODUCTION}

The band gap of a periodic solid containing $N$ electrons per unit cell, formally expressed as the difference between the ionization potential and the electron affinity of the $N$-electron system, fundamentally defines the electrical and optical properties of the material. In practice, the extended nature of the solid system makes it difficult to calculate the band gap using the formal definition, and the band gap is commonly evaluated as the difference between the lowest unoccupied and highest occupied one-electron energies. Eigenvalues obtained with Kohn-Sham (KS) density functional theory (DFT) [1] are intrinsically deficient in describing the band gap because the functional derivative discontinuity of the exchange-correlation (xc) energy is not accounted for by definition [2,3]. As a result, DFT with (semi)local xc functionals underestimates the fundamental band gap by about 50\% [4,5], whereas it falsely predicts a vanishing band gap for certain systems (e.g., Ge) [5].

Realistic band gaps can be achieved by hybrid density functionals incorporating a fraction of orbital-dependent Fock exchange [6]. Implemented within the generalized KS (gKS) scheme [7], the band gap obtained with the eigenvalues of hybrid-functional calculations can be directly interpreted as the fundamental band gap $[3,8]$. Meanwhile, the incorporated Fock exchange mitigates the self-interaction error (viz. delocalization error) prevalent in approximate xc functionals [9], which further contributes to improved band gaps as well as to more accurate lattice parameters and atomization energies [10]. Hence, hybrid functionals have been widely applied to the problems of point defects in solids [11-13] and of band alignments at interfaces [14-18].

Central to the performance of a hybrid functional is the mixing parameter, which determines the fraction of Fock exchange that is admixed with (semi)local DFT exchange. In the early days of hybrid functionals, the mixing parameter was obtained semiempirically to fit experimental atomization energies and ionization potentials. Becke's B3LYP [19], popular in computational chemistry since the early 1990's, admixes $20 \%$ Fock exchange. Later, Perdew et al. proposed a mixing of $\frac{1}{4}$ Fock exchange in view of fourth-order perturbation theory [20]. The use of a fixed mixing parameter, however, does not warrant the universal applicability of the hybrid functional. For example, while the standard PBEH (or PBE0) hybrid functional [21] incorporating 25\% Fock exchange gives realistic band gaps and lattice parameters for semiconductors with moderate band gaps, it becomes inadequate for either wide band-gap insulators (e.g., $\mathrm{MgO}$ and $\mathrm{LiF}$ ) or narrow band-gap semiconductors (e.g., $\mathrm{Si}, \mathrm{Ge}$, and GaAs) [5]. In practice, the mixing parameter often results from an empirical tuning procedure to ensure the validity of the hybrid functional. Notwithstanding, ambiguity on the choice of mixing parameters could arise if the material is less known from experiment. A nonempirical determination of the mixing parameter is therefore critical to exploit the full potential of the hybrid functional, in particular in the context of automated computational material discovery.

Alkauskas et al. [11] and Marques et al. [22] were among the first to identify the connection between the fraction of Fock exchange $\alpha$ and the inverse macroscopic dielectric constant $\varepsilon_{\infty}^{-1}$. Narrow band-gap semiconductors typically show a strong electronic screening and thus need a small $\alpha$, whereas the required value is much larger for wide band-gap insulators. The dielectric dependence of $\alpha$ has already been implied in the screened-exchange (sX) method of Bylander and Kleinman [23] and has been first utilized for band-structure calculations by Shimazaki and Asai [24-26], where $\alpha$ was determined by a model dielectric function [27]. In principle, the dielectric constant can be obtained accurately from first principles by, e.g., finite electric fields $[28,29]$ or linear response theory [3033]. This has been pursued by Koller et al. [34] using a Yukawa screened hybrid functional, and by Skone et al. [35], Ferrari et al. [36], and Gerosa et al. [37] in the form of global hybrid functionals. The resulting dielectric-dependent hybrid (DDH) functionals, primarily the global ones where $\alpha=\varepsilon_{\infty}^{-1}$, have since been used to describe defects [38-42], band alignments of semiconductors [17], and electronic structures of aqueous solutions $[43,44]$. 
A more general form of hybrid functionals rests on the idea of range separation [45], so that the fraction of Fock exchange $\alpha$ is no more a constant but rather range dependent. The idea of range separation was first applied to molecular systems [46] where the asymptotic long-range behavior is critical for describing orbital energies and charge transfer [47]. Depending on the mixing parameters, the Fock exchange can be enhanced either in the short range [48] or in the long range $[49,50]$. Compared to the global hybrid functional, the range-separated version offers more flexibility, but at the same time a nonempirical determination of the mixing parameters is less straightforward [51]. For molecular systems, an optimal tuning scheme for the mixing parameter based on the idea of range separation was proposed by enforcing the Koopmans' condition $[52,53]$. The scheme was later adapted to extended systems [54,55]. In the more recent range-separated DDH (RS-DDH) of Skone et al. [56], the screening length of Fock exchange is determined through the dielectric function calculated from first principles. Yet, the fraction of Fock exchange in RS-DDH is pinned at $25 \%$ in the short range on an empirical basis.

In this work, we present a nonempirical range-separated hybrid functional, of which the mixing parameters are determined solely from $a b$ initio dielectric-function calculations, thereby refraining from using any empirical mixing parameters. This hybrid functional attains reliable electronic structures and lattice parameters for a wide variety of semiconductors and insulators. In particular, we provide a detailed comparison to the RS-DDH hybrid functional, which is closely related to the present method but with reduced Fock exchange in the short range.

The paper is organized as follows. We derive the generalized expression of range-separated hybrid functional with dielectric dependence and describe the computational details in Sec. II. We then show in Sec. III numerical applications to the electronic structure and structural properties for a variety of semiconductors and insulators. In Sec. IV, we remark the general aspects of the hybrid functional and discuss further simplifications towards its use in high-throughput material discovery. We conclude with Sec. V.

\section{METHODS}

\section{A. Generalized range-separated hybrid functional with dielectric dependence}

We start with the general form of range separation of the Coulomb interaction $1 / r_{12}$ as introduced in the Coulombattenuating method (CAM) [57]

$$
\frac{1}{r_{12}}=\underbrace{\frac{a+b \operatorname{erf}\left(\mu r_{12}\right)}{r_{12}}}_{\text {Fock exchange }}+\underbrace{\frac{1-\left[a+b \operatorname{erf}\left(\mu r_{12}\right)\right]}{r_{12}}}_{\text {DFT exchange }},
$$

where $a$ and $a+b$ denote the fraction of the short-range and of the long-range parts of Fock exchange, respectively. The inverse screening length $\mu$ determines the range separation via the error function. Standard hybrid functionals can be recovered by the CAM. For instance, the Heyd-ScuseriaErnzerhof (HSE) screened hybrid functional [48] is obtained by setting $a=0.25, b=-0.25$, and $\mu=0.106 \mathrm{bohr}^{-1}$. The long-range corrected (LC) hybrid functional corresponds to the CAM with $a=0$ and $b=1[49,50]$.

It is our intention to determine the three adjustable parameters, i.e., $a, b$, and $\mu$, from the dielectric function alone without a priori knowledge from experiment. To this end, we write the range-separated Coulomb interaction of Eq. (1) in reciprocal space as the Fourier transform

$$
\frac{1}{\Omega} \frac{4 \pi}{G^{2}}=\frac{1}{\Omega}\{\underbrace{\frac{4 \pi}{G^{2}} \varepsilon^{-1}(G)}_{\text {Fock exchange }}+\underbrace{\frac{4 \pi}{G^{2}}\left[1-\varepsilon^{-1}(G)\right]}_{\text {DFT exchange }}\},
$$

where $\Omega$ is the volume of the unit cell, and the model dielectric function reads as

$$
\varepsilon^{-1}(G)=a+b \cdot e^{-G^{2} / 4 \mu^{2}} .
$$

In effect, the bare Coulomb potential in Eq. (2) is decomposed into a screened Coulomb potential and a polarization term to be described by semilocal DFT exchange. For the HSE hybrid functional where $a=-b=0.25$, the model dielectric function Eq. (3) reduces to $\left(1-e^{-G^{2} / 4 \mu^{2}}\right) / 4$, as previously shown in Ref. [12].

To put the model dielectric function into a more general perspective, we note that, at large wave vectors $G \gg 2 \mu, \varepsilon^{-1}(G)$ approaches unity, leading to $a=1$. In the long-wavelength limit $G=0, \varepsilon^{-1}(0)=\varepsilon_{\infty}^{-1}$ corresponds to the fraction of the long-range component of Fock exchange. Hence, $b$ is given by $\varepsilon_{\infty}^{-1}-1$. The model dielectric function can thus be expressed as

$$
\varepsilon^{-1}(G)=1-\left(1-\varepsilon_{\infty}^{-1}\right) e^{-G^{2} / 4 \mu^{2}} .
$$

Together with the Coulomb potential of Eq. (2), we arrive at the dielectric-dependent range-separated hybrid functional based on the CAM (hereafter denoted as DD-RSH-CAM), of which the range-separated exchange potential is given by

$$
\begin{aligned}
v_{x}\left(\mathbf{r}, \mathbf{r}^{\prime}\right)= & {\left[1-\left(1-\varepsilon_{\infty}^{-1}\right) \operatorname{erf}\left(\mu\left|\mathbf{r}-\mathbf{r}^{\prime}\right|\right)\right] v_{x}^{\text {Fock }}\left(\mathbf{r}, \mathbf{r}^{\prime}\right) } \\
& +\left(1-\varepsilon_{\infty}^{-1}\right) v_{x}^{\mathrm{KS}, \mathrm{lr}}(\mathbf{r}, \mu),
\end{aligned}
$$

where $v_{x}^{\mathrm{KS}, \operatorname{lr}}(\mathbf{r}, \mu)$ refers to the long-range component of the KS potential. The range-separation parameter $\mu$ can be obtained through standard least-squares fitting to the dielectric function $\varepsilon_{\mathbf{G}, \mathbf{G}}^{-1}(\mathbf{q} \rightarrow 0, \omega=0)$ in the long-wavelength limit, as done in Ref. [56]. The inverse macroscopic dielectric constant $\varepsilon_{\infty}^{-1}$ corresponds to the $\mathbf{G}=0$ component of the dielectric function. Since the dielectric function can be calculated from first principles, the DD-RSH-CAM hybrid functional is in principle free of empirical parameters.

The DD-RSH-CAM can be considered as an alternative version of the RS-DDH given that the only difference between the two schemes lies in the description of the short-range exchange. This is illustrated in Fig. 1 where the fraction of Fock exchange is plotted as a function of interelectronic distance. In the short range, the present DD-RSH-CAM prescribes the full Fock exchange as in the sX method $[23,58]$, while the RS-DDH empirically fixes the fraction of Fock exchange at 0.25 [56]. In the long range, both the DD-RSH-CAM and the RS-DDH show a nonvanishing fraction of Fock exchange, which correctly approaches the inverse dielectric constant [59]. 


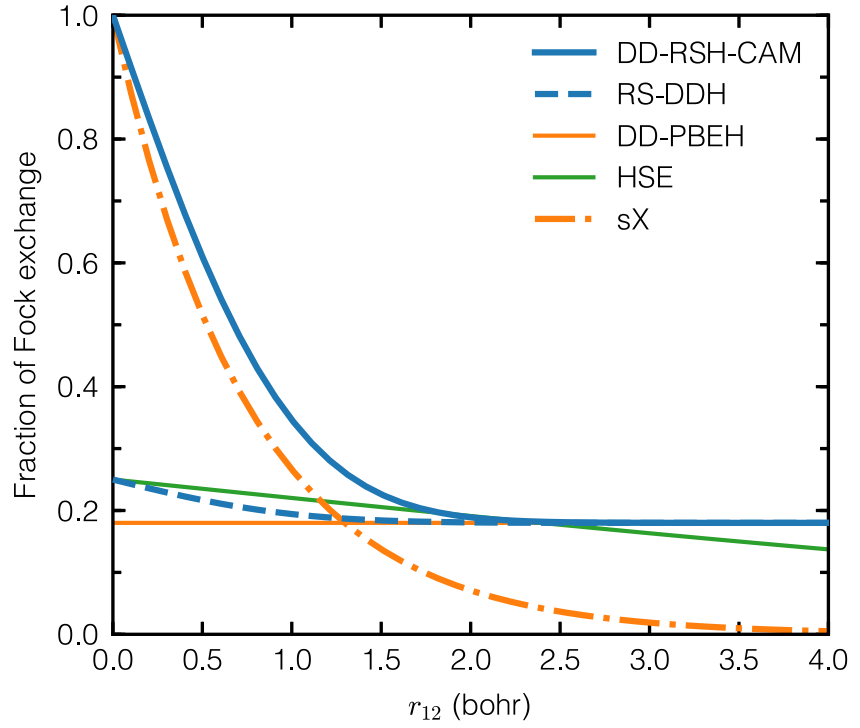

FIG. 1. Fraction of Fock exchange vs interelectronic distance $r_{12}$ in various hybrid-functional schemes. For illustration purpose, we use the macroscopic dielectric constant of diamond (5.6) and an inverse screening length of $0.9 \mathrm{bohr}^{-1}$ (where relevant) for the DDHs, and a Thomas-Fermi wave vector $k_{\mathrm{TF}}$ of $1.3 \mathrm{bohr}^{-1}$ for the screenedexchange (sX) hybrid functional [60]. The HSE uses the standard parameters.

\section{B. Dielectric function calculations}

We follow linear response theory and calculate the dielectric function in terms of the reducible polarizability $\chi$,

$$
\varepsilon_{\mathbf{G}, \mathbf{G}^{\prime}}^{-1}(\mathbf{q}, \omega)=\delta_{\mathbf{G}, \mathbf{G}^{\prime}}(\mathbf{q}, \omega)+v_{\mathbf{G}} \chi_{\mathbf{G}, \mathbf{G}^{\prime}}(\mathbf{q}, \omega),
$$

where $v$ is the bare Coulomb potential. The reducible polarizability relates to the irreducible polarizability $\chi^{0}$ through the Dyson equation

$$
\chi=\chi^{0}+\chi^{0}\left(v+f_{\mathrm{xc}}\right) \chi,
$$

where $f_{\mathrm{xc}}$ is the xc kernel. The irreducible polarizability, known as the noninteracting density-density response function, can be calculated by the formula of Adler and Wiser [61,62] in terms of the gKS orbitals and eigenvalues.

Local-field effects in $\chi$ are included via $v$ and $f_{\mathrm{xc}}$. Neglecting $f_{\mathrm{xc}}$ in Eq. (7) leads to the random phase approximation (RPA) for the reducible polarizability. This is usually a good approximation to calculate $\varepsilon_{\infty}^{-1}$ of semiconductors at the (semi)local KS level [63], but merely as the consequence of the compensation between the too small KS band gap and the missing electron-hole interactions. In the presence of a more realistic band gap (e.g., from hybrid-functional or $G W$ calculations), RPA systematically underestimates the dielectric constant, and the inclusion of a nonlocal $f_{\mathrm{xc}}$ becomes important $[63,64]$. Here, we adopt the parameter-free $f_{\mathrm{xc}}$ given by the bootstrap approximation of Sharma et al. [65]:

$$
f_{\mathrm{xc}, \mathbf{G G}^{\prime}}(\mathbf{q}, \omega)=\frac{v_{\mathbf{G}}^{1 / 2}(\mathbf{q}) \varepsilon_{\mathbf{G G}^{\prime}}^{-1}(\mathbf{q}, 0) v_{\mathbf{G}^{\prime}}^{1 / 2}(\mathbf{q})}{1-\varepsilon_{\mathbf{0 0}}^{\mathrm{RPA}}(\mathbf{q}, 0)} .
$$
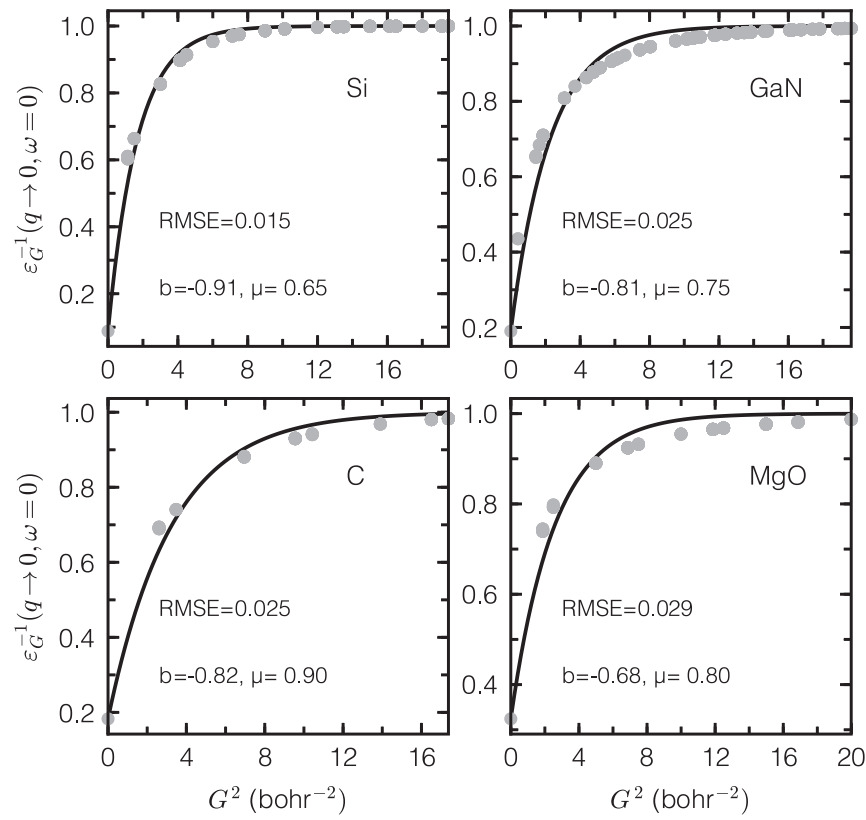

FIG. 2. Calculated dielectric function $\varepsilon^{-1}(\mathbf{q} \rightarrow 0, \omega=0)$ (filled circles) fitted by the model function (solid line). The dielectric function and the DD-RSH-CAM eigenvalues are iterated towards self-consistency. The fitted values and the root-mean-square error (RMSE) are given.

The $\varepsilon_{\mathbf{0 0}}^{\mathrm{RPA}}$ in the denominator of Eq. (8) is the head of the RPA dielectric matrix

$$
\varepsilon_{\mathbf{0 0}}^{\mathrm{RPA}}=1-v_{0} \chi_{\mathbf{0 0}}^{0},
$$

where $v_{0}$ is the long-range component of the Coulomb potential. The bootstrap kernel recovers the asymptotic longrange behavior by construction, and it has been shown to account accurately for the electron-hole interactions in the screened interaction, e.g., in the context of self-consistent $G W$ calculations [66].

Figure 2 shows the static dielectric function in the longwavelength limit calculated through Eqs. (6) to (8) for selected semiconductors and insulators. The dielectric functions are well described by the model function of Eq. (4). The macroscopic dielectric constant, given by the inverse of the $G=0$ component of $\varepsilon^{-1}$, agrees well with experiment (see Sec. III for a detailed comparison with experiment).

\section{Computational details}

The DD-RSH-CAM hybrid functional has been implemented in the ABINIT [89] and QUANTUM ESPRESSO [90] software suites. Specifically, electronic structures and dielectric functions are calculated with ABINIT (a development branch based on v8), whereas structural properties are determined through total-energy calculations with QUANTUM ESPRESSO (a private branch based on v6.2). The recently developed optimized norm-conserving Vanderbilt pseudopotentials (ONCVPSP) $[91,92]$ are used throughout. Semicore states are included for alkali (earth) metals and (post-)transition metals. Table I lists the kinetic plane-wave cutoff energy and the k-point meshes [93], which are chosen to ensure that both 
TABLE I. Plane-wave cutoff energies and $\mathbf{k}$-point meshes used for the calculations. All $k$-point meshes are $\Gamma$ centered unless otherwise specified.

\begin{tabular}{|c|c|c|}
\hline & Cutoff energy (Ry) & k-point mesh \\
\hline $\mathrm{Al}_{2} \mathrm{O}_{3}$ & 90 & $8 \times 8 \times 8$ \\
\hline AlAs & 85 & $12 \times 12 \times 12$ \\
\hline AlN & 85 & $8 \times 8 \times 8$ \\
\hline AlP & 85 & $12 \times 12 \times 12$ \\
\hline $\mathrm{Ar}$ & 60 & $8 \times 8 \times 8$ \\
\hline $\mathrm{BN}$ & 85 & $10 \times 10 \times 10$ \\
\hline $\mathrm{BP}$ & 85 & $12 \times 12 \times 12$ \\
\hline $\mathrm{C}$ & 90 & $12 \times 12 \times 12$ \\
\hline $\mathrm{CaO}$ & 90 & $10 \times 10 \times 10$ \\
\hline $\mathrm{CdS}$ & 100 & $10 \times 10 \times 10$ \\
\hline $\mathrm{CdSe}$ & 120 & $10 \times 10 \times 10$ \\
\hline $\mathrm{Cu}_{2} \mathrm{O}$ & 90 & $10 \times 10 \times 10$ \\
\hline $\mathrm{GaAs}$ & 230 & $12 \times 12 \times 12$ \\
\hline $\mathrm{GaN}$ & 112 & $10 \times 10 \times 10$ \\
\hline $\mathrm{GaP}$ & 112 & $10 \times 10 \times 10$ \\
\hline $\mathrm{Ge}$ & 125 & $12 \times 12 \times 12^{\mathrm{a}}$ \\
\hline $\mathrm{In}_{2} \mathrm{O}_{3}$ & 90 & $6 \times 6 \times 6$ \\
\hline $\mathrm{InP}$ & 90 & $12 \times 12 \times 12$ \\
\hline $\mathrm{LiCl}$ & 100 & $8 \times 8 \times 8$ \\
\hline $\mathrm{LiF}$ & 100 & $8 \times 8 \times 8$ \\
\hline $\mathrm{MgO}$ & 90 & $8 \times 8 \times 8$ \\
\hline $\mathrm{MoS}_{2}$ & 80 & $12 \times 12 \times 12$ \\
\hline $\mathrm{NaCl}$ & 85 & $8 \times 8 \times 8$ \\
\hline $\mathrm{NiO}$ & 100 & $10 \times 10 \times 10$ \\
\hline $\mathrm{Si}$ & 32 & $12 \times 12 \times 12$ \\
\hline $\mathrm{SiC}$ & 90 & $12 \times 12 \times 12$ \\
\hline $\mathrm{SiO}_{2}$ & 90 & $8 \times 8 \times 8$ \\
\hline $\mathrm{SnO}_{2}$ & 100 & $11 \times 11 \times 11$ \\
\hline $\mathrm{TiO}_{2}$ & 90 & $10 \times 10 \times 10$ \\
\hline $\mathrm{ZnO}$ & 110 & $10 \times 10 \times 10$ \\
\hline $\mathrm{ZnS}$ & 110 & $12 \times 12 \times 12$ \\
\hline $\mathrm{ZnSe}$ & 110 & $12 \times 12 \times 12$ \\
\hline
\end{tabular}

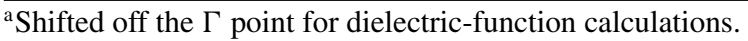

the dielectric constants and the band gaps are well converged. The Coulomb singularity is treated with the Gygi-Baldereschi technique [94]. The evaluation of the polarizability $\chi_{0}$ includes up to 400 unoccupied states and $\mathbf{G}$ vectors with an energy cutoff of 20 Ry.

The selected 32 solid systems span a wide range of band gaps. They include simple $s p$ semiconductors, semiconductors with shallow $d$ states, transition-metal compounds, and ionic insulators. The experimental lattice parameters shown in Table II are used for the electronic structure calculations. The equilibrium lattice constant $a_{0}$ and bulk modulus $B_{0}$ are obtained through a series of total-energy calculations at varying volumes, which are then least-squares fitted to the Rose-Vinet equation of state [95].

The present DD-RSH-CAM hybrid functional uses the PBE $\mathrm{xc}$ functional for the local part of the exchange and correlation. The range-separated PBE exchange is based on the analytical HJS exchange hole of Henderson, Janesko, and Scuseria [96]. We confirm the numerical accuracy of our implementation by obtaining HSE band gaps in accord with previous all-electron and projector-augmented wave calculations [97,98].
TABLE II. Space group and lattice parameters $\left(a_{0}\right.$ and $\left.c_{0} / a_{0}\right)$ of the 32 semiconductors and insulators. Zero-point expansion corrections are not included for the experimental lattice parameters.

\begin{tabular}{|c|c|c|c|c|}
\hline & Space group & $a_{0}(\AA)$ & $c_{0} / a_{0}$ & Reference \\
\hline $\mathrm{Al}_{2} \mathrm{O}_{3}$ & $R \overline{3} c$ & 4.760 & 2.729 & [67] \\
\hline AlAs & $F \overline{4} 3 m$ & 5.661 & & [68] \\
\hline $\mathrm{AlN}$ & $P 6_{3} m c$ & 3.110 & 1.601 & [69] \\
\hline $\mathrm{AlP}$ & $F \overline{4} 3 m$ & 5.451 & & [70] \\
\hline $\mathrm{Ar}$ & $F m \overline{3} m$ & 5.26 & & [71] \\
\hline $\mathrm{BN}$ & $F \overline{4} 3 m$ & 3.616 & & [72] \\
\hline $\mathrm{BP}$ & $F \overline{4} 3 m$ & 4.538 & & [73] \\
\hline $\mathrm{C}$ & $F d \overline{3} m$ & 3.567 & & [74] \\
\hline $\mathrm{CaO}$ & $F m \overline{3} m$ & 4.803 & & [75] \\
\hline $\mathrm{CdS}$ & $F \overline{4} 3 m$ & 5.818 & & [76] \\
\hline $\mathrm{CdSe}$ & $F \overline{4} 3 m$ & 6.052 & & [77] \\
\hline $\mathrm{Cu}_{2} \mathrm{O}$ & $P n \overline{3} m$ & 4.269 & & [78] \\
\hline GaAs & $F \overline{4} 3 m$ & 5.648 & & [79] \\
\hline $\mathrm{GaN}$ & $P 6_{3} m c$ & 3.189 & 1.626 & [80] \\
\hline $\mathrm{GaP}$ & $F \overline{4} 3 m$ & 5.448 & & [79] \\
\hline $\mathrm{Ge}$ & $F d \overline{3} m$ & 5.658 & & [74] \\
\hline $\mathrm{In}_{2} \mathrm{O}_{3}$ & $\operatorname{Ia} \overline{3}$ & 10.117 & & [81] \\
\hline $\mathrm{InP}$ & $F \overline{4} 3 m$ & 5.866 & & [79] \\
\hline $\mathrm{LiCl}$ & $F m \overline{3} m$ & 5.106 & & [79] \\
\hline $\mathrm{LiF}$ & $F m \overline{3} m$ & 4.010 & & [79] \\
\hline $\mathrm{MgO}$ & $F m \overline{3} m$ & 4.207 & & [75] \\
\hline $\mathrm{MoS}_{2}$ & $P 6_{3} / m m c$ & 3.150 & 3.905 & [82] \\
\hline $\mathrm{NaCl}$ & $F m \overline{3} m$ & 5.595 & & [79] \\
\hline $\mathrm{NiO}$ & $F m \overline{3} m$ & 4.170 & & [83] \\
\hline $\mathrm{Si}$ & $F d \overline{3} m$ & 5.430 & & [74] \\
\hline $\mathrm{SiC}$ & $F \overline{4} 3 m$ & 4.358 & & [79] \\
\hline $\mathrm{SiO}_{2}$ & $P 3_{1} 21$ & 4.921 & 1.097 & [84] \\
\hline $\mathrm{SnO}_{2}$ & $P 4_{2} / m n m$ & 4.737 & 0.672 & [85] \\
\hline $\mathrm{TiO}_{2}$ & $\mathrm{P}_{2} / \mathrm{mnm}$ & 4.593 & 0.644 & [86] \\
\hline $\mathrm{ZnO}$ & $\mathrm{Pb}_{3} m c$ & 3.250 & 1.601 & [87] \\
\hline $\mathrm{ZnS}$ & $F \overline{4} 3 m$ & 5.410 & & [88] \\
\hline $\mathrm{ZnSe}$ & $F \overline{4} 3 m$ & 5.667 & & [87] \\
\hline
\end{tabular}

The standard DD-RSH-CAM iterates the dielectric function toward self-consistency along with the wave functions and eigenvalues. We further evaluate a simplified scheme DD0$\mathrm{RSH}-\mathrm{CAM}$, in which the dielectric function is determined in one shot (hence the DD0 prefix) at the RPA level (i.e., with $f_{x c}=0$ ) on top of PBE eigenvalues. The mixing parameters obtained from the RPA-PBE dielectric function are then used in the CAM hybrid-functional calculations without further iterations.

Table III summarizes the mixing parameters of the hybrid functionals to be assessed in this work. In addition to the DD-RSH-CAM, we include for comparison the standard HSE, the global dielectric-dependent PBEH hybrid functional (DDPBEH), and the RS-DDH. The DD-PBEH is characterized by a range-independent mixing parameter given by the inverse dielectric constant (cf. Fig. 1). The RS-DDH differs from the DD-RSH-CAM in that the fraction of Fock exchange is reduced to 0.25 in the short range. As described in Ref. [56], such a choice is made on empirical grounds and the attenuation of the Fock exchange in practice accounts for some short-range correlation in the functional. By comparing the DD-RSH-CAM 
TABLE III. List of hybrid functionals considered in this work. The mixing parameters $(a, b$, and $\mu)$ follow the convention of the CAM hybrid functional given in Eq. (1). $\varepsilon_{\infty}^{\mathrm{RPA}}$ refers to the RPA macroscopic dielectric constant calculated in one shot on top of the PBE starting point, whereas $\varepsilon_{\infty}^{\mathrm{RPA}+f_{\mathrm{xc}}}$ is evaluated self-consistently with the respective dielectric-dependent hybrid functional. The global DD-PBEH can be obtained with a vanishing $b$ or $\mu$.

\begin{tabular}{lccc}
\hline \hline & $a$ & $b$ & $\mu\left(\mathrm{bohr}^{-1}\right)$ \\
\hline HSE & 0.25 & -0.25 & 0.106 \\
DD-PBEH & $\left(\varepsilon_{\infty}^{\mathrm{RPA}+f_{\mathrm{xc}}}\right)^{-1}$ & 0 & $(0)$ \\
RS-DDH & 0.25 & $\left(\varepsilon_{\infty}^{\mathrm{RPA}+f_{\mathrm{xc}}}\right)^{-1}-0.25$ & Table IV \\
DD-RSH-CAM & 1 & $\left(\varepsilon_{\infty}^{\mathrm{RPA}+f_{\mathrm{xc}}}\right)^{-1}-1$ & Table IV \\
DD0-RSH-CAM & 1 & $\left(\varepsilon_{\infty}^{\mathrm{RPA}}\right)^{-1}-1$ & Table IV \\
\hline \hline
\end{tabular}

to the RS-DDH, this work therefore also sheds light on the role of the short-range Fock exchange in the RSH.

All calculations are realized with the CAM hybrid functional using the corresponding mixing parameters shown in Table III. While formally equivalent, we note that the dielectric functions underlying the present RS-DDH and the one originally proposed in Ref. [56] are calculated differently. In particular, the long-range nonlocal contribution is taken into account by the bootstrap kernel in this work, whereas in Ref. [56] it is achieved directly from the nonlocal xc potentials. Nevertheless, the dielectric constants and screening parameters obtained with the two approaches agree well in general (cf. Sec. III A).

\section{RESULTS}

\section{A. Macroscopic dielectric constant and screening parameter}

Table IV shows the macroscopic dielectric constants $\varepsilon_{\infty}$ of the 32 solid systems determined either by the RPA at the PBE level, or self-consistently by the full expression including the bootstrap xc kernel (RPA $+f_{\mathrm{xc}}$ ) at the hybrid-functional level. For simple $s p$ semiconductors and insulators, the RPA-PBE dielectric constants are close to the experimental values. The agreement worsens in the presence of semicore $d$ electrons in the valence band. The more rigorous $\mathrm{RPA}+f_{\mathrm{xc}}$ treatment on top of the dielectric-dependent hybrid functionals improves the description of the dielectric constants. In particular, the MAPE is reduced to $5 \%$ when calculated with DD-RSH-CAM. Nevertheless, the discrepancy is largely diminished in the inverse dielectric constant $\varepsilon_{\infty}^{-1}$, as all the methods show an MAE of 0.02 or smaller.

The screening parameter, i.e., the inverse screening length $\mu$, shows a much smaller variation across the various materials. Specifically, we find an average value of $0.71 \mathrm{bohr}^{-1}$ and a standard deviation of $0.07 \mathrm{bohr}^{-1}$ for the $\mu$ given in Table IV.

\section{B. Band gap}

We now assess the band gaps obtained by the DD-RSHCAM hybrid functional. To consolidate the conclusion about the accuracy, we preferably compare to experimental fundamental band gaps measured at (or extrapolated to) low temperature. Spin-orbit splitting at the VBM is included $a$ posteriori for AlAs, GaAs, Ge, CdSe, and ZnSe [118], which accounts for a reduction of about $0.1 \mathrm{eV}$ in the band gap. Since our calculations do not consider electron-phonon coupling, the zero-phonon renormalization (ZPR) of the band gap needs to be subtracted from the calculated band gap when one compares theoretical to experimental band gaps. In particular, for polar materials, the long-range Fröhlich part of the electron-phonon self-energy cannot be neglected [129,146]. For ionic insulators, the recent calculation of Lambrecht et al. shows band-gap renormalizations up to $0.5 \mathrm{eV}$ alone from Fröhlich electronphonon coupling [129]. Altogether, the electron-phonon coupling can lead to sizable ZPRs, for instance of over $1 \mathrm{eV}$ for $\mathrm{LiF}$ [131]. In this work, we take into account the Fröhlich polaronic coupling for $\mathrm{MgO}, \mathrm{NaCl}, \mathrm{LiCl}$, and $\mathrm{LiF}$, using the results of Refs. [129,131].

The calculated band gaps are presented in Table $\mathrm{V}$ and are displayed versus experimental band gaps in Fig. 3. The DD-RSH-CAM shows accurate band gaps for the majority of the compounds, including a wide variety of group IV semiconductors ( $\mathrm{Si}, \mathrm{C}, \mathrm{SiC}$ ), II-VI semiconductors ( $\mathrm{ZnSe}$, $\mathrm{ZnO}, \mathrm{CdS}, \mathrm{CdSe}$ ), III-V semiconductors (AlAs, AlN, AlP, $\mathrm{GaAs}, \mathrm{GaN}, \mathrm{GaP}, \mathrm{InP})$, oxides $\left(\mathrm{CaO}, \mathrm{Cu}_{2} \mathrm{O}, \mathrm{NiO}, \mathrm{MgO}\right)$, and alkali halides $(\mathrm{NaCl}, \mathrm{LiCl}, \mathrm{LiF})$. The associated mean signed error (MSE) is $0.09 \mathrm{eV}$ and the mean absolute error (MAE) is $0.23 \mathrm{eV}$, to be compared to the MAE of $0.44 \mathrm{eV}$ by one-shot $G_{0} W_{0}$ [147] and of $0.13 \mathrm{eV}$ by state-of-the-art self-consistent $G W$ calculations [66]. For some of the oxides, such as $\mathrm{Al}_{2} \mathrm{O}_{3}$, $\mathrm{In}_{2} \mathrm{O}_{3}$, and $\mathrm{SiO}_{2}$, the overestimation of the band gaps are more pronounced with the DD-RSH-CAM. Another notable outlier is $\mathrm{TiO}_{2}$, for which the calculated band gap is over $20 \%$ off. We note that the Fröhlich polaron coupling, which has hitherto not yet been estimated for $\mathrm{TiO}_{2}$, can be significant as a result of the strong polaronic effect [148]. This is supported by a recent $G W$ calculation showing a band gap of $3.7 \mathrm{eV}$ for rutile $\mathrm{TiO}_{2}$ [66].

The overall accuracy of DD-RSH-CAM is maintained with the simplified DD0-RSH-CAM scheme, in accord with the good agreement for $\varepsilon_{\infty}^{-1}$ shown in Sec. III A. On the other hand, while the global DD-PBEH is adequate for describing the band gaps of simple $s p$ semiconductors and insulators, it produces too small band gaps for some transition metal and post-transition metal compounds, such as $\mathrm{Ge}, \mathrm{GaAs}, \mathrm{Cu}_{2} \mathrm{O}$, $\mathrm{NiO}$, and a series of $\mathrm{Cd} X$ and $\mathrm{Zn} X$ compounds. This is to some extent related to the underestimated dielectric constant for these materials (cf. Sec. III A), but we note that even the use of experimental dielectric constant does not necessarily bring the DD-PBE band gap in full agreement with experiment. The RSDDH marginally increases the band gaps for these compounds compared to the DD-PBEH, but the band gaps remain too small. Among the DDHs, the best agreement is achieved with the $\mathrm{DD}(0)$-CAM owing to the full Fock exchange included in the short range.

In Fig. 3, we correlate the calculated and measured band gaps via linear regression. The predictive power of DD-RSHCAM is clearly manifested by the near-ideal slope of 1.02 and a small intercept of $-0.01 \mathrm{eV}$. The DD-PBEH and RS-DDH show comparable slopes, but the intercepts are more markedly off the origin. Finally, the standard HSE hybrid functional is characterized by a considerably lower slope than those of the DDHs, showing sizable deviations for materials beyond narrow band-gap semiconductors. 
TABLE IV. Macroscopic dielectric constants calculated through the RPA expression at the PBE level and through the full expression including vertex corrections $\left(\mathrm{RPA}+f_{\mathrm{xc}}\right.$ ) with various dielectric-dependent hybrid functionals. The mean signed error (MSE), mean absolute error (MAE), and mean absolute percentage error (MAPE) with respect to experimental dielectric constants are given. The MAE of the inverse dielectric constants is also included. In addition, the inverse screening lengths $\mu$ obtained by least-squares fit to the dielectric functions are given and compared to the values reported in Ref. [56].

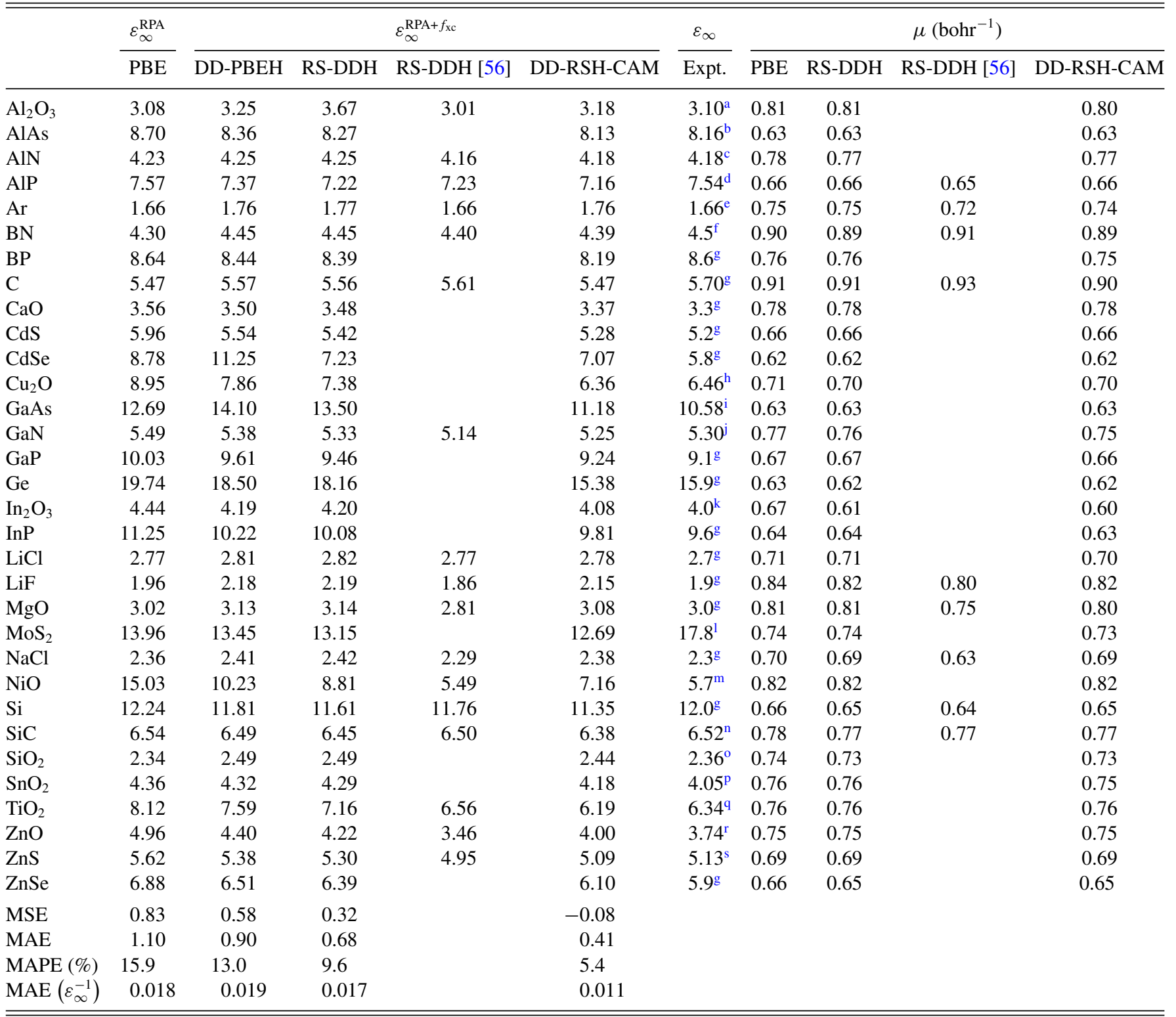

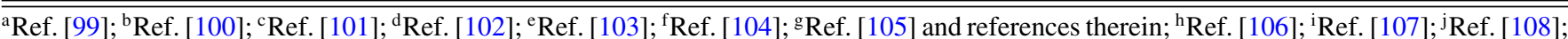
${ }^{\mathrm{k}}$ Ref. [109]; ${ }^{\mathrm{R}}$ Ref. [110]; ${ }^{\mathrm{m}}$ Ref. [111]; ${ }^{\mathrm{n}}$ Ref. [112]; ${ }^{\circ}$ Ref. [113] and references therein; ${ }^{\mathrm{P}}$ Ref. [114]; ${ }^{\mathrm{Q}}$ Ref. [115]; ${ }^{\mathrm{r}}$ Ref. [116]; ${ }^{\mathrm{s}}$ Ref. [117].

It is noteworthy that the present RS-DDH shows a higher error than that reported in Ref. [56]. For a more consistent comparison, we perform the statistics for the common compounds found in both studies and include the ZPR throughout. We find a MAPE of $8.3 \%$ for our calculated RS-DDH band gaps, higher than the MAPE of 5.5\% as reported in Ref. [56]. In particular, our calculated RS-DDH band gaps are apparently smaller than the values given in the reference for a few compounds (such as $\mathrm{Al}_{2} \mathrm{O}_{3}, \mathrm{NiO}, \mathrm{MgO}, \mathrm{LiF}$, and $\mathrm{ZnO}$ ), the origin of which can be associated with the discrepancy present in the dielectric constants (cf. Table IV). Indeed, the agreement among the band gaps can be largely reconciled once the dielectric constants of Ref. [56] are applied to the present
RS-DDH calculations. We speculate that such a discrepancy could result from the use of different basis sets (plane-wave vs Gaussian basis sets), or more likely, from the choice of the $f_{\mathrm{xc}}$ in calculating the $\varepsilon_{\infty}^{-1}$. At variance with the bootstrap kernel, the $f_{\mathrm{xc}}$ in Ref. [56] is evaluated directly as the functional derivative of the xc potential, thereby approximately capturing the excitonic effect through the nonlocal exchange included in the hybrid functional [55,63,149-151]. More calculations, preferably using a more rigorous $f_{\mathrm{xc}}$ kernel [152], are needed to elucidate the efficacy of the approximate $f_{x c}$ kernels for the DDHs. Meanwhile, to exclude the ambiguity arising from the calculated dielectric constant, it is reasonable to use the experimental dielectric constant to determine the fraction of 
TABLE V. Band gaps $E_{\mathrm{g}}$ (in eV) of the 32 semiconductors and insulators calculated by DD-RSH-CAM among other hybrid functionals. Experimental band gaps refer to the low-temperature values. The effect of spin-orbit coupling at the VBM is included for AlAs (0.11), GaAs (0.12), Ge (0.10), CdSe (0.14), and ZnSe (0.14) [118], where the values in parentheses have been subtracted from the calculated band gaps. The zero-phonon renormalization (ZPR) is given if available. The MAPE is calculated by comparing the calculated band gap to $\left(E_{\mathrm{g}}^{\text {expt }}+\mathrm{ZPR}\right)$ and by referring the difference to $E_{\mathrm{g}}^{\text {expt }}$.

\begin{tabular}{|c|c|c|c|c|c|c|c|c|c|}
\hline & PBE & HSE & DD-PBEH & RS-DDH & RS-DDH [56] & DD0-RSH-CAM & DD-RSH-CAM & Expt. & ZPR \\
\hline $\mathrm{Al}_{2} \mathrm{O}_{3}$ & 6.27 & 8.07 & 9.40 & 8.98 & 9.61 & 9.60 & 9.51 & $9.1^{\mathrm{a}}$ & \\
\hline AlAs & 1.32 & 1.93 & 1.91 & 1.90 & & 2.05 & 2.11 & $2.24^{\mathrm{b}}$ & $0.04^{\mathrm{c}}$ \\
\hline AlN & 4.18 & 5.49 & 6.10 & 6.11 & 6.23 & 6.25 & 6.26 & $6.23^{\mathrm{b}}$ & $0.24^{\mathrm{c}}$ \\
\hline AlP & 1.56 & 2.27 & 2.30 & 2.33 & 2.40 & 2.41 & 2.52 & $2.52^{\mathrm{b}}$ & $0.02^{\mathrm{c}}$ \\
\hline $\mathrm{Ar}$ & 8.70 & 10.36 & 14.31 & 14.02 & 14.41 & 14.94 & 14.67 & $14.3^{\mathrm{d}}$ & \\
\hline $\mathrm{BN}$ & 4.53 & 5.83 & 6.37 & 6.39 & 6.33 & 6.55 & 6.56 & $6.4^{\mathrm{e}}$ & $0.34^{\mathrm{f}}$ \\
\hline $\mathrm{BP}$ & 1.25 & 1.99 & 1.92 & 1.93 & & 2.02 & 2.02 & $2.1^{\mathrm{g}}$ & \\
\hline $\mathrm{C}$ & 4.21 & 5.35 & 5.56 & 5.57 & 5.43 & 5.63 & 5.61 & $5.48^{\mathrm{h}}$ & $0.37^{\mathrm{c}}$ \\
\hline $\mathrm{CaO}$ & 3.66 & 5.30 & 6.42 & 6.39 & & 6.99 & 7.17 & $7.09^{\mathrm{i}}$ & \\
\hline $\mathrm{CdS}$ & 1.16 & 2.19 & 2.37 & 2.42 & & 2.81 & 2.95 & $2.58^{c}$ & $0.06^{\mathrm{c}}$ \\
\hline $\mathrm{CdSe}$ & 0.49 & 1.37 & 1.11 & 1.33 & & 1.56 & 1.75 & $1.85^{\mathrm{c}}$ & $0.03^{\mathrm{c}}$ \\
\hline $\mathrm{Cu}_{2} \mathrm{O}$ & 0.49 & 1.89 & 1.52 & 1.71 & & 2.06 & 2.49 & $2.17^{\mathrm{c}}$ & $0.03^{c}$ \\
\hline GaAs & 0.42 & 1.26 & 0.79 & 0.90 & & 1.38 & 1.45 & $1.52^{\mathrm{b}}$ & $0.05^{\mathrm{c}}$ \\
\hline $\mathrm{GaN}$ & 1.94 & 3.14 & 3.32 & 3.34 & 3.30 & 3.43 & 3.50 & $3.51^{\mathrm{b}}$ & $0.17^{\mathrm{c}}$ \\
\hline $\mathrm{GaP}$ & 1.65 & 2.31 & 2.15 & 2.19 & & 2.36 & 2.42 & $2.35^{\mathrm{b}}$ & $0.08^{c}$ \\
\hline $\mathrm{Ge}$ & 0.00 & 0.61 & 0.18 & 0.22 & & 0.34 & 0.50 & $0.74^{\mathrm{b}}$ & $0.05^{\mathrm{c}}$ \\
\hline $\mathrm{In}_{2} \mathrm{O}_{3}$ & 1.21 & 2.50 & 3.16 & 3.06 & & 3.29 & 3.51 & $2.93^{\mathrm{j}}$ & \\
\hline InP & 0.69 & 1.47 & 1.21 & 1.25 & & 1.49 & 1.55 & $1.42^{\mathrm{b}}$ & $0.05^{\mathrm{c}}$ \\
\hline $\mathrm{LiCl}$ & 6.41 & 7.80 & 9.49 & 9.41 & 9.54 & 9.87 & 9.89 & $9.4^{\mathrm{k}}$ & $0.17^{1}$ \\
\hline $\mathrm{LiF}$ & 9.27 & 11.50 & 14.90 & 14.59 & 15.42 & 15.97 & 15.56 & $14.2^{\mathrm{m}}$ & $1.15^{\mathrm{n}}$ \\
\hline $\mathrm{MgO}$ & 4.80 & 6.47 & 7.93 & 7.88 & 8.27 & 8.28 & 8.19 & $7.83^{\mathrm{i}}$ & $0.53^{n}$ \\
\hline $\mathrm{MoS}_{2}$ & 0.88 & 1.42 & 1.23 & 1.16 & & 1.35 & 1.40 & $1.29^{\circ}$ & \\
\hline $\mathrm{NaCl}$ & 5.20 & 6.56 & 8.75 & 8.64 & 8.71 & 9.10 & 9.10 & $8.97^{p}$ & $0.17^{1}$ \\
\hline $\mathrm{NiO}$ & 1.02 & 4.23 & 2.67 & 3.32 & 4.51 & 4.05 & 4.68 & $4.3^{\mathrm{q}}$ & \\
\hline $\mathrm{Si}$ & 0.70 & 1.14 & 0.96 & 0.99 & 1.01 & 1.10 & 1.14 & $1.17^{\mathrm{r}}$ & $0.06^{\mathrm{c}}$ \\
\hline $\mathrm{SiC}$ & 1.36 & 2.24 & 2.33 & 2.35 & 2.31 & 2.41 & 2.47 & $2.42^{\mathrm{s}}$ & $0.11^{\mathrm{t}}$ \\
\hline $\mathrm{SiO}_{2}$ & 5.95 & 7.69 & 9.99 & 9.88 & & 10.58 & 10.40 & $9.7^{\mathrm{u}}$ & \\
\hline $\mathrm{SnO}_{2}$ & 1.29 & 2.96 & 3.49 & 3.59 & & 3.95 & 3.99 & $3.60^{\mathrm{v}}$ & \\
\hline $\mathrm{TiO}_{2}$ & 1.92 & 3.39 & 3.06 & 3.25 & 3.17 & 3.84 & 4.18 & $3.3^{\mathrm{w}}$ & $0.15^{x}$ \\
\hline $\mathrm{ZnO}$ & 0.85 & 2.43 & 2.94 & 3.02 & 3.67 & 3.33 & 3.74 & $3.44^{y}$ & $0.16^{\mathrm{c}}$ \\
\hline $\mathrm{ZnS}$ & 2.10 & 3.29 & 3.46 & 3.53 & 3.86 & 4.01 & 4.08 & $3.84^{z}$ & $0.10^{\mathrm{c}}$ \\
\hline $\mathrm{ZnSe}$ & 1.19 & 2.20 & 2.12 & 2.19 & & 2.59 & 2.64 & $2.82^{z}$ & $0.05^{\mathrm{c}}$ \\
\hline MSE & -2.14 & -0.89 & -0.37 & -0.35 & & 0.02 & 0.09 & & \\
\hline MAE & 2.14 & 0.89 & 0.42 & 0.37 & & 0.25 & 0.23 & & \\
\hline MAPE (\%) & 51.1 & 16.2 & 15.6 & 13.3 & & 7.9 & 7.3 & & \\
\hline
\end{tabular}

${ }^{\mathrm{a}}$ Ref. [119], optical band gap; ${ }^{\mathrm{b}}$ Ref. [80]; ${ }^{\mathrm{c}}$ Ref. [120]; ${ }^{\mathrm{d}}$ Ref. [121]; ${ }^{\mathrm{e}}$ Ref. [122], optical band gap at $300 \mathrm{~K}$; ${ }^{\mathrm{f}}$ Ref. [123]; ${ }^{\mathrm{g}}$ Ref. [124], optical band gap at room temperature; ${ }^{\text {h}}$ Ref. [125], optical band gap at $300 \mathrm{~K} ;{ }^{\mathrm{i}}$ Ref. [126]; ${ }^{\mathrm{j}}$ Ref. [127]; ${ }^{\mathrm{k}}$ Ref. [128]; ${ }^{\mathrm{l}}$ Fröhlich polaronic renormalization taken from Ref. [129]; ${ }^{m}$ Ref. [130]; ${ }^{n}$ Ref. [131], total ZPR including Fröhlich polaronic renormalization. Similar results can be obtained by combining the polaronic renormalization estimated in Ref. [129] (MgO: $0.22 \mathrm{eV}$; LiF: $0.48 \mathrm{eV}$ ) with the ZPR excluding the long-range Fröhlich part (MgO: $0.27 \mathrm{eV}$; LiF: $0.46 \mathrm{eV}$ ) reported in Ref. [123]; ${ }^{\circ}$ Ref. [132]; ${ }^{\mathrm{p}}$ Ref. [133]. Another widely cited value is $8.5 \mathrm{eV}$ (cf. Ref. [134]); ${ }^{\mathrm{q}} \mathrm{Ref}$. [135]. Antiferromagnetic ordering is assumed; ${ }^{\mathrm{r}} \operatorname{Ref}$. [136]; ${ }^{\mathrm{s}} \mathrm{Ref}$. [137]; ${ }^{\mathrm{t}}$ Ref. [138]; " Indirect fundamental gap deduced from the experimental absorption spectra (Ref. [139]) and the calculated excitonic binding energy of $1.2 \mathrm{eV}$ (Ref. [140]); ${ }^{\mathrm{v}} \operatorname{Ref} .[141]$; ${ }^{\mathrm{w}} \operatorname{Ref}$. [142]; ${ }^{\mathrm{x}} \operatorname{Ref}$. [143]; ${ }^{\mathrm{y}} \operatorname{Ref}$. [144]; ${ }^{\text {Ref. [145]. }}$

the long-range Fock exchange in the DD-RSH-CAM. This modifies the band gaps by no more than $0.1 \mathrm{eV}$ on average, although more pronounced increases $(0.3-0.6 \mathrm{eV})$ can be observed in the case of $\mathrm{NiO}$ and of the two wide band-gap materials, i.e., Ar and LiF.

\section{Valence band structure}

The localized semicore $d$ states are often too shallow in the valence band in (semi)local DFT owing to the delocalization error. Such an issue can be mitigated by hybrid functionals, but the correct description of the $d$ band depends on the fraction of the incorporated Fock exchange. In Table VI, the position of the $d$ band is examined for II-VI and III-V semiconductors using the hybrid functionals. The DD-RSH-CAM and DD0RSH-CAM again show good agreement with experiment. In fact, they tend to slightly overbind the $d$ electrons. The HSE with standard mixing parameters is insufficient to recover the experimental $d$-band positions, placing them about $1.4 \mathrm{eV}$ too 

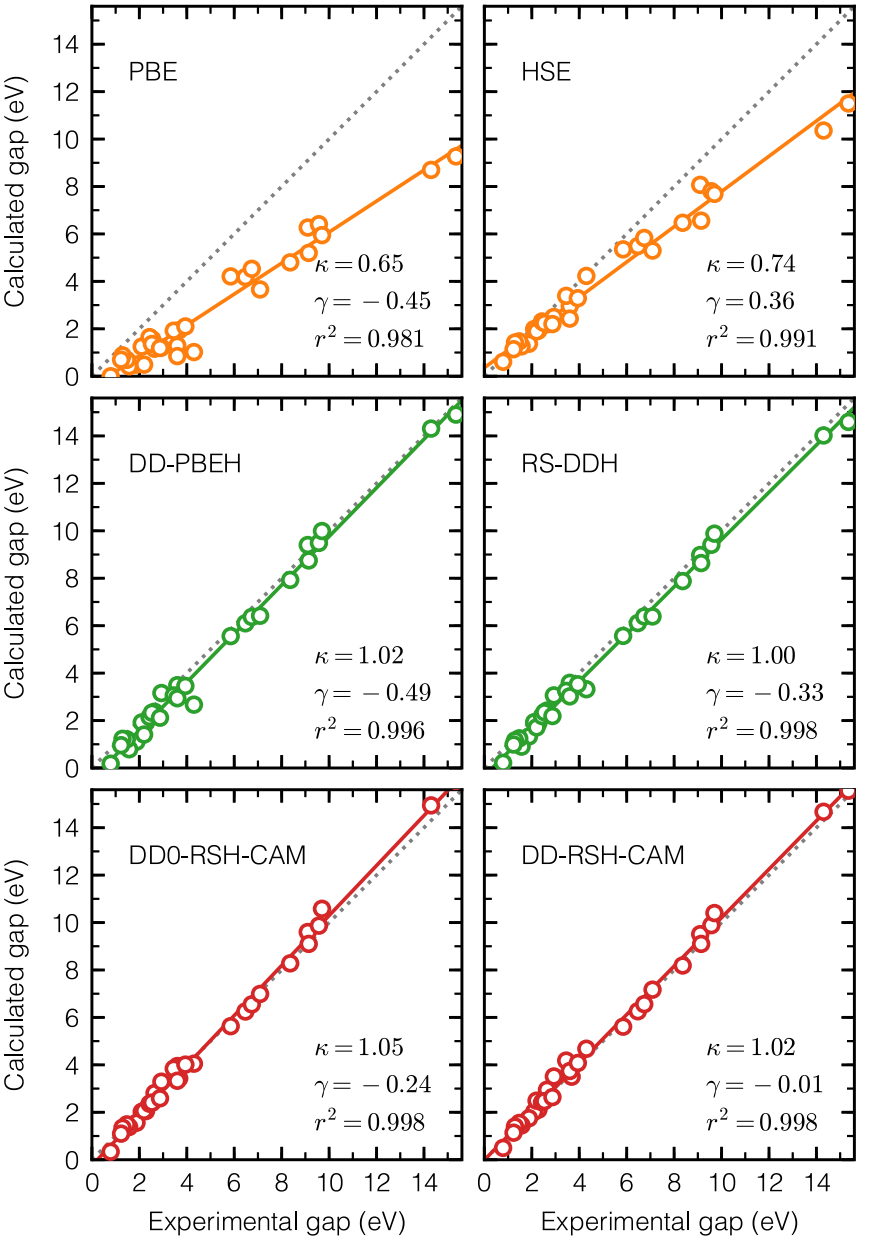

FIG. 3. Calculated band gaps $\left(E_{\mathrm{g}}^{\text {calc }}\right)$ plotted against (ZPR corrected) experimental reference values $\left(E_{\mathrm{g}}^{\text {expt }}\right)$. The solid lines are linear regressions given by $E_{\mathrm{g}}^{\text {calc }}=\kappa E_{\mathrm{g}}^{\text {exp }}+\gamma$. The slope $\kappa$, intercept $\gamma$ (in $\mathrm{eV}$ ), and correlation coefficient $r^{2}$ are given. The dashed lines refer to the ideal cases corresponding to $E_{\mathrm{g}}^{\mathrm{calc}}=E_{\mathrm{g}}^{\mathrm{expt}}$. shallow on average. The global DD-PBEH leads to the least accurate binding energies for the semicore $d$ states, whereas the binding energies given by the RS-DDH are still too shallow, in compliance with the fact that the DD-PBEH and RS-DDH tend to underestimate the band gaps for these materials.

We further assess how the valence bandwidth is reproduced by the hybrid functionals. As seen in Table VII, all the hybrid functionals account well for the valence bandwidth of the selected compounds. Overall, we find that iterating the dielectric function in DD-RSH-CAM hardly affects the valence bandwidth and the binding energy of semicore $d$ states compared to the one-shot method.

\section{Structural properties}

Moving to the structural properties, we calculate the equilibrium lattice constant $\left(a_{0}\right)$ and bulk modulus $\left(B_{0}\right)$ for some representative semiconductors and insulators. For simplicity, the mixing parameters ( $b$ and $\mu$ ) are kept fixed at the values obtained with the experimental volume. The optimal mixing parameters could actually vary depending on the actual volume used in the series of total-energy calculations. Such an effect is found to be small for DDHs [34,56], and is considered to be negligible for DD-RSH-CAM where the dependence only involves the long-range part of Fock exchange.

As shown in Table VIII, the present DD-RSH-CAM yields significantly more realistic lattice constants and bulk moduli compared to the semilocal PBE functional. The structural properties are markedly accurate for the $s p$ semiconductors. We do not consider DD0-RSH-CAM, as the results achieved with this functional would essentially agree with DD-RSH-CAM, as for the electronic structures discussed earlier. Admittedly, the lattice constants and bulk moduli are already of good accuracy with standard hybrid functionals, such as HSE [97] (see also Table VIII), or with some semilocal functionals [169]. Nevertheless, we show here that a good description of the electronic and structural properties can be obtained concurrently across a wide variety of materials with the DDRSH-CAM scheme.

TABLE VI. Average positions of the occupied $d$ band $(\mathrm{eV})$ relative to the VBM for the II-V and III-V semiconductors.

\begin{tabular}{|c|c|c|c|c|c|c|}
\hline & HSE & DD-PBEH & RS-DDH & DD0-RSH-CAM & DD-RSH-CAM & Expt. \\
\hline $\mathrm{CdS}$ & -8.5 & -8.2 & -8.3 & -9.6 & -9.7 & $-9.6^{\mathrm{a}}$ \\
\hline $\mathrm{CdSe}$ & -8.9 & -8.2 & -8.6 & -10.1 & -10.2 & $-10.0^{\mathrm{a}}$ \\
\hline $\mathrm{InP}$ & -15.8 & -14.8 & -15.2 & -17.2 & -17.3 & $-16.8^{\mathrm{a}}$ \\
\hline GaAs & -17.3 & -15.5 & -16.4 & -20.5 & -20.6 & $-18.9^{b}$ \\
\hline $\mathrm{GaN}$ & -15.3 & -14.8 & -15.1 & -17.9 & -18.0 & $-17.0^{\mathrm{c}}$ \\
\hline $\mathrm{GaP}$ & -16.8 & -15.5 & -16.1 & -19.6 & -19.7 & $-18.7^{\mathrm{a}}$ \\
\hline $\mathrm{ZnO}$ & -6.0 & -5.9 & -6.0 & -7.3 & -7.5 & $-7.5^{\mathrm{d}}$ \\
\hline $\mathrm{ZnS}$ & -7.5 & -7.1 & -7.3 & -9.5 & -9.5 & $-9.0^{\mathrm{a}}$ \\
\hline $\mathrm{ZnSe}$ & -8.0 & -7.3 & -7.6 & -10.1 & -10.2 & $-9.2^{\mathrm{a}}$ \\
\hline MSE & 1.4 & 2.2 & 1.8 & -0.6 & -0.7 & \\
\hline MAE & 1.4 & 2.2 & 1.8 & 0.6 & 0.7 & \\
\hline MAPE $(\%)$ & 11.9 & 17.3 & 14.6 & 4.4 & 4.7 & \\
\hline
\end{tabular}

\footnotetext{
${ }^{\mathrm{a}}$ Reference [153].

${ }^{\mathrm{b}}$ Reference [154].

${ }^{\mathrm{c}}$ Reference [155].

${ }^{\mathrm{d}}$ Reference [156].
} 
TABLE VII. Calculated valence bandwidths (eV) compared to experiment.

\begin{tabular}{|c|c|c|c|c|c|c|}
\hline & HSE & DD-PBEH & RS-DDH & DD0-RSH-CAM & DD-RSH-CAM & Expt. \\
\hline $\mathrm{Al}_{2} \mathrm{O}_{3}$ & 6.8 & 7.0 & 6.9 & 7.2 & 7.2 & $7^{\mathrm{a}}$ \\
\hline $\mathrm{BN}$ & 22.1 & 22.0 & 22.0 & 23.0 & 23.1 & $22.0^{\mathrm{b}}$ \\
\hline $\mathrm{C}$ & 23.6 & 23.0 & 23.1 & 24.0 & 24.0 & $23.0^{\mathrm{c}}$ \\
\hline $\mathrm{Ge}$ & 14.1 & 13.1 & 13.3 & 13.8 & 13.9 & $12.9^{\mathrm{d}}$ \\
\hline $\mathrm{LiF}$ & 3.2 & 3.3 & 3.3 & 3.3 & 3.2 & $3.5^{\mathrm{e}}$ \\
\hline $\mathrm{MgO}$ & 4.9 & 5.0 & 5.0 & 5.1 & 5.1 & $5.0^{\mathrm{f}}$ \\
\hline $\mathrm{Si}$ & 13.3 & 12.5 & 12.6 & 12.9 & 13.0 & $12.5^{\mathrm{g}}$ \\
\hline $\mathrm{SiC}$ & 17.0 & 16.4 & 16.5 & 17.1 & 17.2 & 16.9 \\
\hline $\mathrm{ZnO}$ & 6.8 & 6.8 & 6.8 & 8.0 & 8.1 & $9.0^{\mathrm{g}}$ \\
\hline MSE & 0.0 & -0.3 & -0.3 & 0.3 & 0.3 & \\
\hline MAE & 0.6 & 0.3 & 0.4 & 0.6 & 0.6 & \\
\hline MAPE (\%) & 6.4 & 3.9 & 4.3 & 4.7 & 5.1 & \\
\hline
\end{tabular}

${ }^{\text {a Reference [119]. }}$

${ }^{\mathrm{b}}$ Reference [122].

${ }^{\mathrm{c}}$ Reference [157].

${ }^{\mathrm{d}}$ Reference [158].

${ }^{\mathrm{e}}$ Reference [159].

${ }^{\mathrm{f}}$ Reference [160].

${ }^{\mathrm{g}}$ Reference [161] and references therein.

\section{DISCUSSION}

\section{A. General remarks on range-separated DDHs}

We have seen in Sec. II A that, by retaining the diagonal matrix elements of the dielectric function $\varepsilon_{\mathbf{G G}}^{-1}$, the DDH naturally attains the form of range separation. Such a DDH, namely the DD-RSH-CAM, recovers the full Fock exchange in the short range while maintaining the desired asymptotic $\varepsilon_{\infty}^{-1} r^{-1}$ decay in the long range. Both features turn out to be essential for a DDH. The long-range contribution is predominant in achieving a realistic band structure. To show this, we exclude the Fock exchange in the long range by setting $a=-b=1$ in Eq. (1). The obtained transition energy gaps shown in Table IX are strongly underestimated compared to the full DD-RSH-CAM. In this case, a much larger screening length (4-5 bohr vs $1-2$ bohr) would be required [173], effectively compensating the

TABLE VIII. Lattice constant $\left(a_{0}\right)$ and bulk modulus $\left(B_{0}\right)$ for a subset of compounds. The experimental lattice constants are corrected for zero-point anharmonic expansion (ZPAE) [162].

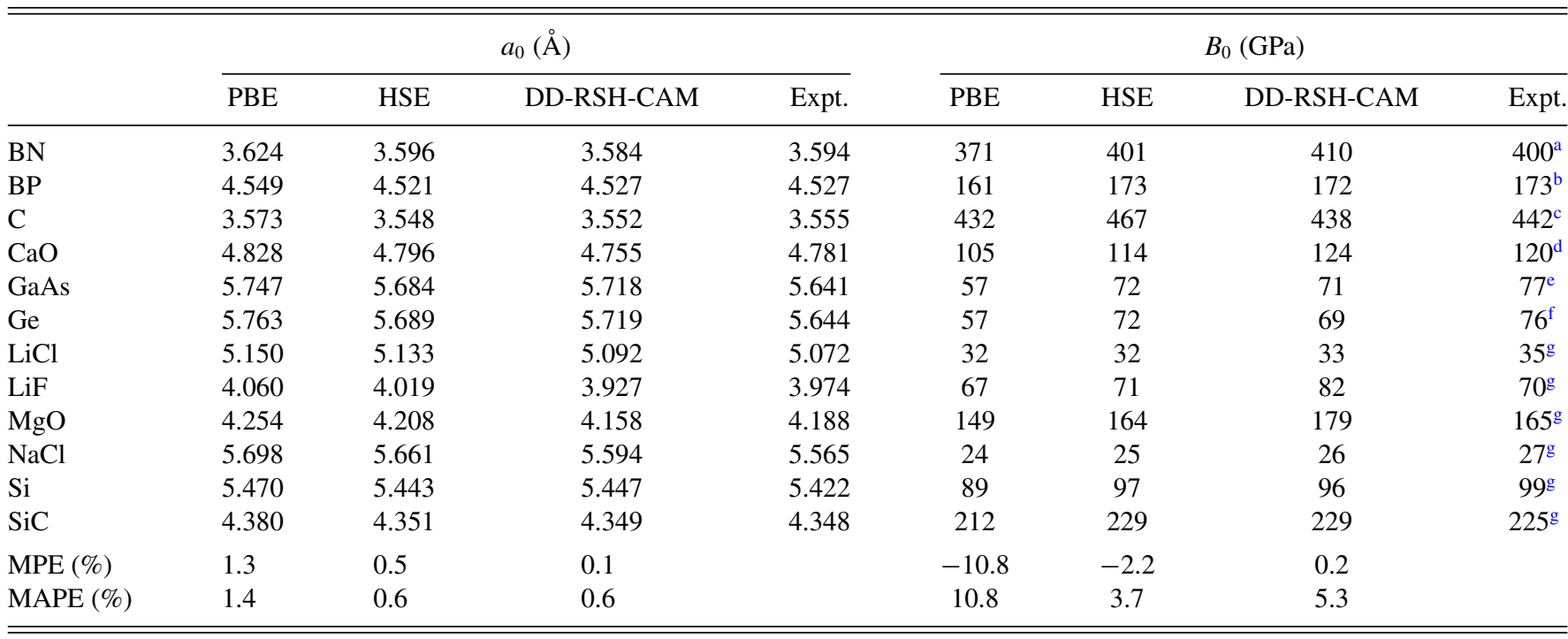

\footnotetext{
${ }^{\mathrm{a}}$ Reference [163].

${ }^{\mathrm{b}}$ Reference [164].

${ }^{\mathrm{c}}$ Reference [165].

${ }^{\mathrm{d}}$ Reference [166], extrapolated to $0 \mathrm{~K}$.

${ }^{\mathrm{e}}$ Reference [167].

${ }^{\mathrm{f}}$ Reference [168].

g Reference [169].
} 
TABLE IX. Comparison of transition energy gaps calculated with DD-RSH-CAM, DD-RSH-CAM with vanishing long-range Fock exchange $(b=-1)$, and RS-DDH (DD-RSH-CAM with $\frac{1}{4}$ of Fock exchange in the short range). The fraction of long-range Fock exchange and the screening length use the ones of the standard DD-RSH-CAM throughout. The percentage error (PE) with respect to experiment is given next to the band gap $E_{g}$. When multiple transitions are present, the same ZPR is applied to the calculated band gaps to estimate the PE.

\begin{tabular}{|c|c|c|c|c|c|c|c|c|c|}
\hline & & \multicolumn{2}{|c|}{ DD-RSH-CAM } & \multicolumn{2}{|c|}{ DD-RSH-CAM $(b=-1)$} & \multicolumn{2}{|c|}{ RS-DDH } & \multirow{2}{*}{$\frac{\text { Expt. }}{E_{g}(\mathrm{eV})}$} & \multirow[b]{2}{*}{$\mathrm{ZPR}$} \\
\hline & & $E_{g}(\mathrm{eV})$ & $\operatorname{PE}(\%)$ & $E_{g}(\mathrm{eV})$ & $\mathrm{PE}(\%)$ & $E_{g}(\mathrm{eV})$ & $\mathrm{PE}(\%)$ & & \\
\hline \multirow[t]{3}{*}{$\mathrm{Si}$} & $\Gamma \rightarrow \Gamma$ & 3.29 & -5 & 2.80 & -19 & 3.09 & -11 & $3.4^{\mathrm{a}}$ & \multirow[t]{3}{*}{0.06} \\
\hline & $\Gamma \rightarrow X$ & 1.29 & -2 & 0.86 & -36 & 1.17 & -11 & $1.25^{\mathrm{b}}$ & \\
\hline & $\Gamma \rightarrow L$ & 2.20 & -11 & 1.73 & -30 & 2.04 & -17 & $2.4^{\mathrm{a}}$ & \\
\hline \multirow[t]{3}{*}{ GaAs } & $\Gamma \rightarrow \Gamma$ & 1.45 & -8 & 0.96 & -40 & 1.02 & -36 & 1.52 & \multirow[t]{3}{*}{0.05} \\
\hline & $\Gamma \rightarrow X$ & 1.93 & -14 & 1.73 & -23 & 1.80 & -20 & $2.18^{\mathrm{a}}$ & \\
\hline & $\Gamma \rightarrow L$ & 1.75 & -8 & 1.50 & -21 & 1.44 & -24 & $1.84^{\mathrm{a}}$ & \\
\hline $\mathrm{ZnO}$ & $\Gamma \rightarrow \Gamma$ & 3.74 & 4 & 1.72 & -55 & 3.20 & -12 & 3.44 & 0.16 \\
\hline $\mathrm{C}$ & $\Gamma \rightarrow \Gamma$ & 7.45 & -3 & 6.00 & -23 & 7.17 & -7 & $7.3^{c}$ & 0.37 \\
\hline $\mathrm{MgO}$ & $\Gamma \rightarrow \Gamma$ & 8.19 & -2 & 5.08 & -42 & 7.92 & -6 & 7.83 & 0.53 \\
\hline
\end{tabular}

${ }^{\mathrm{a}}$ Reference [170].

${ }^{\mathrm{b}}$ Reference [171].

${ }^{\mathrm{c}}$ Reference [172].

missing long-range contribution. Regarding the short-range contribution, we compare DD-RSH-CAM to the RS-DDH in Table IX. The importance of the unscreened Fock exchange in the short range is evident as the RS-DDH energy gaps are generally more underestimated than the DD-RSH-CAM ones. The effect is particularly pronounced for GaAs, as the RS-DDH fails to recover the experimental band gap by a large margin. Indeed, the band gap of GaAs, Ge, and some II-VI semiconductors (e.g., $\mathrm{Cd} X$ and $\operatorname{Zn} X$ ) with localized semicore $d$ states are commonly underestimated by DDHs [17]. We show that the issue can be addressed, to a large extent, by the enhanced short-range Fock exchange as included in the DD-RSH-CAM.

We have mentioned in Sec. III B that the DD-RSH-CAM is prone to overestimate the band gaps for some wide band-gap oxides, including $\mathrm{Al}_{2} \mathrm{O}_{3}$ and $\mathrm{SiO}_{2}$. The calculated dielectric constants are nevertheless in reasonable agreement with experiment. In fact, since the global DD-PBEH already gives too high band gaps for these oxides, it is foreseeable that the enhanced Fock exchange in the short range would inevitably worsens the agreement with experiment. On the other hand, by fixing the short-range Fock exchange to $\frac{1}{4}$, the RS-DDH effectively mitigates the band-gap overestimation with the DD-PBEH for these two oxides, provided that their dielectric constants are below 4. To see if such an empirical choice of the short-range Fock exchange is globally more justified, we assess the accuracy of DD-RSH-CAM and RS-DDH separately on two groups of materials, one with the dielectric constant over 4 and the other below 4 . The results are summarized in Table X. In particular, our calculations reveal that the full Fock exchange in the short range consistently leads to a more accurate description of the band gaps.

It is intuitive to compare the present DD-RSH-CAM scheme to the Coulomb-hole and screened-exchange (COHSEX) approximation to the self-energy operator [174]. The screened exchange of the present DD-RSH-CAM rests on the dielectric function derived from the diagonal part of the static dielectric matrix. The local-field and dynamic effects are thus neglected in the screened exchange, akin to the static diagonal-COHSEX method [94]. Nonetheless, these effects are insignificant for homogeneous systems and the resulting screened exchange is sufficiently accurate for bulk solids [175]. The other contribution, namely the Coulomb hole, is effectively accounted for by a fraction of semilocal PBE exchange [cf. the second term in Eq. (2)] plus the full PBE correlation. Such a semilocal description turns out to be adequate in light of the high accuracy attained by the DD-RSH-CAM.

\section{B. Practical aspects}

While the full self-consistent DD-RSH-CAM sets the benchmark accuracy that the functional can attain, in practice one can dismiss the cumbersome iterations of the dielectric functions and rather rely upon the RPA-PBE dielectric function without much loss of accuracy. At variance with other DDHs, the role of the dielectric constant is weakened by the unscreened Fock exchange used in the short range.

In a more aggressive move, the dielectric constant can be determined by a simple model function so that the entire dielectric function calculations are no longer needed, recovering the computational cost of a standard hybrid functional. As a proof of concept, we use the following Penn model [27]:

$$
\varepsilon_{\infty}^{\mathrm{Penn}}=1+\left(\frac{\omega_{p}}{\bar{E}_{g}^{\mathrm{HSE}}}\right)^{2}\left(1-\frac{\bar{E}_{g}^{\mathrm{HSE}}}{4 E_{F}}\right)
$$

TABLE X. Band-gap errors for the two groups of materials classified according to the experimental dielectric constant. Group I contains 23 compounds, and group II contains 9 compounds.

\begin{tabular}{lcccccc}
\hline \hline & \multicolumn{2}{c}{$\mathrm{I}\left(\varepsilon_{\infty} \geq 4\right)$} & & \multicolumn{2}{c}{$\mathrm{II}\left(\varepsilon_{\infty}<4\right)$} \\
\cline { 2 - 3 } \cline { 5 - 6 } & DD-RSH-CAM & RS-DDH & & DD-RSH-CAM & RS-DDH \\
\hline MSE $(\mathrm{eV})$ & 0.04 & -0.34 & & 0.22 & -0.38 \\
MAE $(\mathrm{eV})$ & 0.22 & 0.35 & & 0.27 & 0.42 \\
MAPE $(\%)$ & 8.9 & 16.2 & & 3.0 & 5.6 \\
\hline \hline
\end{tabular}


TABLE XI. Validation of the DD0-RSH-CAM method using dielectric constants calculated with the Penn model given by Eq. (10). A fixed inverse screening length of $0.71 \mathrm{bohr}^{-1}$ is used.

\begin{tabular}{lcccc}
\hline \hline & $E_{g}(\mathrm{eV})$ & $\mathrm{PE}\left(E_{g}\right)(\%)$ & $\varepsilon_{\infty}^{\text {Penn }}$ & $\mathrm{PE}\left(\varepsilon_{\infty}^{\text {Penn }}\right)(\%)$ \\
\hline $\mathrm{AlAs}$ & 2.03 & -11.1 & 8.7 & 6.2 \\
$\mathrm{AlP}$ & 2.38 & -6.3 & 8.5 & 12.2 \\
$\mathrm{BN}$ & 6.42 & -5.0 & 5.5 & 21.8 \\
$\mathrm{C}$ & 5.70 & -2.7 & 6.0 & 6.0 \\
$\mathrm{CdS}$ & 2.76 & 4.6 & 5.8 & 11.7 \\
$\mathrm{CdSe}$ & 1.73 & -8.1 & 6.2 & 6.72 \\
$\mathrm{GaAs}$ & 1.47 & -6.6 & 9.2 & -13.1 \\
$\mathrm{GaP}$ & 2.41 & -0.8 & 8.7 & -4.2 \\
$\mathrm{Ge}$ & 0.43 & -48.6 & 11.1 & -30.4 \\
$\mathrm{InP}$ & 1.58 & 7.7 & 8.4 & -13.0 \\
$\mathrm{Si}$ & 1.12 & -8.5 & 10.8 & -9.6 \\
$\mathrm{SiC}$ & 2.28 & -10.3 & 8.3 & 27.0 \\
$\mathrm{ZnS}$ & 3.80 & -3.6 & 5.9 & 14.6 \\
$\mathrm{ZnSe}$ & 2.44 & -15.2 & 6.3 & 6.6 \\
\hline \hline
\end{tabular}

where $\omega_{p}=\sqrt{4 \pi n_{v}}$ is the plasma frequency as a function of the valence density $n_{v}, \bar{E}_{g}^{\mathrm{HSE}}$ is the HSE energy gap averaged over the Brillouin zone, and $E_{F}=\left(3 \pi^{2} n_{v}\right)^{2 / 3} / 2$ corresponds to the free-electron Fermi energy. The inverse dielectric constants $\varepsilon_{\infty}^{-1}$ calculated from Eq. (10) show an excellent MAE of 0.01 . Concerning the inverse screening length $\mu$, we simply choose its mean value $\left(0.71 \mathrm{bohr}^{-1}\right)$ as it is less material dependent. With these simplifications, we recalculate the band gaps of some semiconductors. Table XI shows that the results are quantitatively reliable, mostly within $10 \%$ of the experimental band gaps, despite the larger deviations in the dielectric constants. Overall, we find that the results are not worse than those calculated with the RPA-PBE dielectric function. Meanwhile, they show a general improvement over the HSE band gaps, reducing the MAPE from $15 \%$ to $10 \%$ for the semiconductors listed in Table XI. For wide band-gap materials, the model-function approach needs to be used with precaution since the long-range part of Fock exchange becomes important and deviations in $\epsilon_{\infty}$ are amplified in the calculated band gaps.

As far as the electronic structure is concerned, the perturbative approach of hybrid-functional calculations is often sufficient provided that the semilocal description of the wave functions is close to that of the hybrid functional (see for instance Refs. [34,176,177]). As such, the computational cost of the DD-RSH-CAM can be further reduced, whereas the effect is typically within $0.1 \mathrm{eV}$ for the band gap $[34,176,177]$. On a more general remark, recent developments have shown that the computational cost of hybrid functionals can be significantly reduced through a compressed representation of the Fock exchange operator [178] or a localized representation of the orbitals $[179,180]$, making the DD-RSH-CAM a feasible method for the purpose of high-throughput materials screening.

\section{CONCLUSION}

We have presented a range-separated hybrid functional of which the mixing parameters are determined solely from the static dielectric function. The resulting DD-RSH-CAM hybrid functional prescribes the full Fock exchange in the short range and maintains the correct asymptotic behavior in the long range. Our calculations show that this hybrid functional successfully reproduces the electronic and structural properties for a variety of semiconductors and insulators without using any tuning parameters. In particular, the enhanced short-range Fock exchange remedies the band-gap problem for certain systems, such as GaAs, which would otherwise show a too small band gap. Numerically, the current method can take advantage of the dielectric constant calculated either in the RPA or even with a simple model function, thereby refraining from iterating the dielectric function and making the method as fast as standard hybrid functionals. Combined with its global accuracy, the nonempirical DD-RSH-CAM is therefore well suited for high-throughput calculations, e.g., in the field of materials discovery and screening.

\section{ACKNOWLEDGMENTS}

We appreciate fruitful discussions with $\mathrm{M}$. Giantomassi on pseudopotentials and with A. Baldereschi and F. Naccarato on model dielectric functions. Support from the Swiss National Science Foundation (SNSF) is acknowledged under Grant No. 200020-172524. The calculations have been performed at the Swiss National Supercomputing Center (CSCS) and at the SCITAS of EPFL. The present research has also benefited from computational resources made available on the Tier-1 supercomputer of the Fédération Wallonie-Bruxelles, infrastructure funded by the Walloon Region under Grant No. 1117545. W.C. and G.-M.R. acknowledge support from the Communauté française de Belgique through the BATTAB project (Project No. RC 14/19-057). G.-M.R. is grateful to the National Fund for Scientific Research (F.R.S.-FNRS) of Belgium for financial support.
[1] W. Kohn and L. J. Sham, Phys. Rev. 140, A1133 (1965).

[2] J. P. Perdew, R. G. Parr, M. Levy, and J. L. Balduz, Phys. Rev. Lett. 49, 1691 (1982).

[3] W. Yang, A. J. Cohen, and P. Mori-Sánchez, J. Chem. Phys. 136, 204111 (2012).

[4] M. Shishkin and G. Kresse, Phys. Rev. B 75, 235102 (2007).

[5] W. Chen and A. Pasquarello, Phys. Rev. B 90, 165133 (2014).
[6] S. Kümmel and L. Kronik, Rev. Mod. Phys. 80, 3 (2008).

[7] A. Seidl, A. Görling, P. Vogl, J. A. Majewski, and M. Levy, Phys. Rev. B 53, 3764 (1996).

[8] J. P. Perdew, W. Yang, K. Burke, Z. Yang, E. K. U. Gross, M. Scheffler, G. E. Scuseria, T. M. Henderson, I. Y. Zhang, A. Ruzsinszky, H. Peng, J. Sun, E. Trushin, and A. Görling, Proc. Natl. Acad. Sci. U.S.A. 114, 2801 (2017).

[9] J. P. Perdew and A. Zunger, Phys. Rev. B 23, 5048 (1981). 
[10] L. Schimka, J. Harl, and G. Kresse, J. Chem. Phys. 134, 024116 (2011).

[11] A. Alkauskas, P. Broqvist, and A. Pasquarello, Phys. Status Solidi. B 248, 775 (2011).

[12] C. Freysoldt, B. Grabowski, T. Hickel, J. Neugebauer, G. Kresse, A. Janotti, and C. G. Van de Walle, Rev. Mod. Phys. 86, 253 (2014).

[13] W. Chen and A. Pasquarello, J. Phys.: Condens. Matter 27, 133202 (2015).

[14] A. Alkauskas, P. Broqvist, F. Devynck, and A. Pasquarello, Phys. Rev. Lett. 101, 106802 (2008).

[15] K. Steiner, W. Chen, and A. Pasquarello, Phys. Rev. B 89, 205309 (2014)

[16] Y. Hinuma, A. Grüneis, G. Kresse, and F. Oba, Phys. Rev. B 90, 155405 (2014).

[17] Y. Hinuma, Y. Kumagai, I. Tanaka, and F. Oba, Phys. Rev. B 95, 075302 (2017).

[18] Z. Guo, F. Ambrosio, W. Chen, P. Gono, and A. Pasquarello, Chem. Mater. 30, 94 (2018).

[19] A. D. Becke, J. Chem. Phys. 98, 5648 (1993).

[20] J. P. Perdew, M. Ernzerhof, and K. Burke, J. Chem. Phys. 105, 9982 (1996).

[21] C. Adamo and V. Barone, J. Chem. Phys. 110, 6158 (1999).

[22] M. A. L. Marques, J. Vidal, M. J. T. Oliveira, L. Reining, and S. Botti, Phys. Rev. B 83, 035119 (2011).

[23] D. M. Bylander and L. Kleinman, Phys. Rev. B 41, 7868 (1990).

[24] T. Shimazaki and Y. Asai, Chem. Phys. Lett. 466, 91 (2008).

[25] T. Shimazaki and Y. Asai, J. Chem. Phys. 130, 164702 (2009).

[26] T. Shimazaki and Y. Asai, J. Chem. Phys. 132, 224105 (2010).

[27] D. R. Penn, Phys. Rev. 128, 2093 (1962).

[28] P. Umari and A. Pasquarello, Phys. Rev. Lett. 89, 157602 (2002).

[29] I. Souza, J. Íñiguez, and D. Vanderbilt, Phys. Rev. Lett. 89, 117602 (2002).

[30] S. Baroni and R. Resta, Phys. Rev. B 33, 7017 (1986).

[31] X. Gonze and C. Lee, Phys. Rev. B 55, 10355 (1997).

[32] S. Baroni, S. de Gironcoli, A. Dal Corso, and P. Giannozzi, Rev. Mod. Phys. 73, 515 (2001).

[33] G. Onida, L. Reining, and A. Rubio, Rev. Mod. Phys. 74, 601 (2002).

[34] D. Koller, P. Blaha, and F. Tran, J. Phys.: Condens. Matter 25, 435503 (2013).

[35] J. H. Skone, M. Govoni, and G. Galli, Phys. Rev. B 89, 195112 (2014).

[36] A. M. Ferrari, R. Orlando, and M. Rérat, J. Chem. Theory Comput. 11, 3245 (2015).

[37] M. Gerosa, C. E. Bottani, L. Caramella, G. Onida, C. Di Valentin, and G. Pacchioni, Phys. Rev. B 91, 155201 (2015).

[38] M. Gerosa, C. D. Valentin, C. E. Bottani, G. Onida, and G. Pacchioni, J. Chem. Phys. 143, 111103 (2015).

[39] M. Gerosa, C. E. Bottani, L. Caramella, G. Onida, C. D. Valentin, and G. Pacchioni, J. Chem. Phys. 143, 134702 (2015).

[40] M. Gerosa, C. Di Valentin, G. Onida, C. E. Bottani, and G. Pacchioni, J. Phys. Chem. C 120, 11716 (2016).

[41] P. Scherpelz and G. Galli, Phys. Rev. Mater. 1, 021602 (2017).

[42] M. Gerosa, C. E. Bottani, C. D. Valentin, G. Onida, and G. Pacchioni, J. Phys.: Condens. Matter 30, 044003 (2018).

[43] A. P. Gaiduk, M. Govoni, R. Seidel, J. H. Skone, B. Winter, and G. Galli, J. Am. Chem. Soc. 138, 6912 (2016).
[44] T. A. Pham, M. Govoni, R. Seidel, S. E. Bradforth, E. Schwegler, and G. Galli, Sci. Adv. 3, e1603210 (2017).

[45] T. Leininger, H. Stoll, H.-J. Werner, and A. Savin, Chem. Phys. Lett. 275, 151 (1997).

[46] H. Iikura, T. Tsuneda, T. Yanai, and K. Hirao, J. Chem. Phys. 115, 3540 (2001).

[47] T. Tsuneda and K. Hirao, WIREs Comput. Mol. Sci. 4, 375 (2014).

[48] J. Heyd, G. E. Scuseria, and M. Ernzerhof, J. Chem. Phys. 118, 8207 (2003); 124, 219906 (2006).

[49] O. A. Vydrov and G. E. Scuseria, J. Chem. Phys. 125, 234109 (2006).

[50] I. C. Gerber, J. G. Ángyán, M. Marsman, and G. Kresse, J. Chem. Phys. 127, 054101 (2007).

[51] J. E. Moussa, P. A. Schultz, and J. R. Chelikowsky, J. Chem. Phys. 136, 204117 (2012).

[52] S. Refaely-Abramson, R. Baer, and L. Kronik, Phys. Rev. B 84, 075144 (2011).

[53] S. Refaely-Abramson, S. Sharifzadeh, N. Govind, J. Autschbach, J. B. Neaton, R. Baer, and L. Kronik, Phys. Rev. Lett. 109, 226405 (2012).

[54] S. Refaely-Abramson, S. Sharifzadeh, M. Jain, R. Baer, J. B. Neaton, and L. Kronik, Phys. Rev. B 88, 081204 (2013).

[55] S. Refaely-Abramson, M. Jain, S. Sharifzadeh, J. B. Neaton, and L. Kronik, Phys. Rev. B 92, 081204 (2015).

[56] J. H. Skone, M. Govoni, and G. Galli, Phys. Rev. B 93, 235106 (2016).

[57] T. Yanai, D. P. Tew, and N. C. Handy, Chem. Phys. Lett. 393, 51 (2004).

[58] S. J. Clark and J. Robertson, Phys. Rev. B 82, 085208 (2010).

[59] V. Fiorentini and A. Baldereschi, Phys. Rev. B 51, 17196 (1995).

[60] Y. Guo, J. Robertson, and S. J. Clark, J. Phys.: Condens. Matter 27, 025501 (2015).

[61] S. L. Adler, Phys. Rev. 126, 413 (1962).

[62] N. Wiser, Phys. Rev. 129, 62 (1963).

[63] J. Paier, M. Marsman, and G. Kresse, Phys. Rev. B 78, 121201 (2008).

[64] M. Shishkin, M. Marsman, and G. Kresse, Phys. Rev. Lett. 99, 246403 (2007).

[65] S. Sharma, J. K. Dewhurst, A. Sanna, and E. K. U. Gross, Phys. Rev. Lett. 107, 186401 (2011).

[66] W. Chen and A. Pasquarello, Phys. Rev. B 92, 041115 (2015).

[67] S. Kondo, K. Tateishi, and N. Ishizawa, Jpn. J. Appl. Phys. 47, 616 (2008).

[68] M. Ettenberg and R. J. Paff, J. Appl. Phys. 41, 3926 (1970).

[69] H. Schulz and K. Thiemann, Solid State Commun. 23, 815 (1977).

[70] A. Addamiano, Acta Crystallogr. 13, 505 (1960).

[71] D. G. Henshaw, Phys. Rev. 111, 1470 (1958).

[72] V. L. Solozhenko, D. Häusermann, M. Mezouar, and M. Kunz, Appl. Phys. Lett. 72, 1691 (1998).

[73] J. A. Perri, S. La Placa, and B. Post, Acta Crystallogr. 11, 310 (1958).

[74] H. Holloway, K. C. Hass, M. A. Tamor, T. R. Anthony, and W. F. Banholzer, Phys. Rev. B 44, 7123 (1991).

[75] D. K. Smith and H. R. Leider, J. Appl. Crystallogr. 1, 246 (1968).

[76] R. J. Traill and R. W. Boyle, Am. Mineral. 40, 555 (1955).

[77] A. Stuckes and G. Farrell, J. Phys. Chem. Solids 25, 477 (1964). 
[78] F. Oba, F. Ernst, Y. Yu, R. Liu, H. M. Kothari, and J. A. Switzer, J. Am. Ceram. Soc. 88, 253 (2005).

[79] P. Haas, F. Tran, and P. Blaha, Phys. Rev. B 79, 085104 (2009).

[80] I. Vurgaftman, J. R. Meyer, and L. R. Ram-Mohan, J. Appl. Phys. 89, 5815 (2001).

[81] M. Marezio, Acta Crystallogr. 20, 723 (1966).

[82] N. Wakabayashi, H. G. Smith, and R. M. Nicklow, Phys. Rev. B 12, 659 (1975).

[83] L. C. Bartel and B. Morosin, Phys. Rev. B 3, 1039 (1971).

[84] H. d'Amour, W. Denner, and H. Schulz, Acta Crystallogr. B 35, 550 (1979).

[85] W. H. Baur, Acta Crystallogr. 9, 515 (1956).

[86] J. K. Burdett, T. Hughbanks, G. J. Miller, J. W. Richardson, and J. V. Smith, J. Am. Chem. Soc. 109, 3639 (1987).

[87] H. Karzel, W. Potzel, M. Köfferlein, W. Schiessl, M. Steiner, U. Hiller, G. M. Kalvius, D. W. Mitchell, T. P. Das, P. Blaha, K. Schwarz, and M. P. Pasternak, Phys. Rev. B 53, 11425 (1996).

[88] M. J. Cooper, K. D. Rouse, and H. Fuess, Acta Crystallogr. A 29, 49 (1973).

[89] X. Gonze, B. Amadon, P.-M. Anglade, J.-M. Beuken, F. Bottin, P. Boulanger, F. Bruneval, D. Caliste, R. Caracas, M. Côté, T. Deutsch, L. Genovese, P. Ghosez, M. Giantomassi, S. Goedecker, D. Hamann, P. Hermet, F. Jollet, G. Jomard, S. Leroux et al., Comput. Phys. Commun. 180, 2582 (2009).

[90] P. Giannozzi, S. Baroni, N. Bonini, M. Calandra, R. Car, C. Cavazzoni, D. Ceresoli, G. L. Chiarotti, M. Cococcioni, I. Dabo, A. Dal Corso, S. de Gironcoli, S. Fabris, G. Fratesi, R. Gebauer, U. Gerstmann, C. Gougoussis, A. Kokalj, M. Lazzeri, L. Martin-Samos et al., J. Phys.: Condens. Matter 21, 395502 (2009).

[91] D. R. Hamann, Phys. Rev. B 88, 085117 (2013); 95, 239906(E) (2017).

[92] The pseudopotenials are available at http://www.pseudodojo.org. The stringent sets are used.

[93] H. J. Monkhorst and J. D. Pack, Phys. Rev. B 13, 5188 (1976).

[94] F. Gygi and A. Baldereschi, Phys. Rev. B 34, 4405 (1986).

[95] P. Vinet, J. R. Smith, J. Ferrante, and J. H. Rose, Phys. Rev. B 35, 1945 (1987).

[96] T. M. Henderson, B. G. Janesko, and G. E. Scuseria, J. Chem. Phys. 128, 194105 (2008).

[97] J. Paier, M. Marsman, K. Hummer, G. Kresse, I. C. Gerber, and J. G. Ángyán, J. Chem. Phys. 124, 154709 (2006); 125, 249901 (2006).

[98] C. Friedrich, M. Betzinger, M. Schlipf, S. Blügel, and A. Schindlmayr, J. Phys.: Condens. Matter 24, 293201 (2012).

[99] R. H. French, H. Müllejans, and D. J. Jones, J. Am. Ceram. Soc. 81, 2549 (1998).

[100] R. E. Fern and A. Onton, J. Appl. Phys. 42, 3499 (1971).

[101] S. Shokhovets, R. Goldhahn, G. Gobsch, S. Piekh, R. Lantier, A. Rizzi, V. Lebedev, and W. Richter, J. Appl. Phys. 94, 307 (2003).

[102] B. Monemar, Solid State Commun. 8, 1295 (1970).

[103] A. C. Sinnock and B. L. Smith, Phys. Rev. 181, 1297 (1969).

[104] P. J. Gielisse, S. S. Mitra, J. N. Plendl, R. D. Griffis, L. C. Mansur, R. Marshall, and E. A. Pascoe, Phys. Rev. 155, 1039 (1967).

[105] J. A. Van Vechten, Phys. Rev. 182, 891 (1969).

[106] M. O’Keeffe, J. Chem. Phys. 39, 1789 (1963).
[107] W. J. Moore and R. T. Holm, J. Appl. Phys. 80, 6939 (1996); 81, 3732 (1997).

[108] M. Giehler, M. Ramsteiner, O. Brandt, H. Yang, and K. H. Ploog, Appl. Phys. Lett. 67, 733 (1995).

[109] I. Hamberg and C. G. Granqvist, J. Appl. Phys. 60, R123 (1986).

[110] A. R. Beal and H. P. Hughes, J. Phys. C: Solid State Phys. 12, 881 (1979).

[111] P. J. Gielisse, J. N. Plendl, L. C. Mansur, R. Marshall, S. S. Mitra, R. Mykolajewycz, and A. Smakula, J. Appl. Phys. 36, 2446 (1965).

[112] L. Patrick and W. J. Choyke, Phys. Rev. B 2, 2255 (1970).

[113] C. T. Kirk, Phys. Rev. B 38, 1255 (1988).

[114] R. Summitt, J. Appl. Phys. 39, 3762 (1968).

[115] J. R. DeVore, J. Opt. Soc. Am. 41, 416 (1951).

[116] N. Ashkenov, B. N. Mbenkum, C. Bundesmann, V. Riede, M. Lorenz, D. Spemann, E. M. Kaidashev, A. Kasic, M. Schubert, M. Grundmann, G. Wagner, H. Neumann, V. Darakchieva, H. Arwin, and B. Monemar, J. Appl. Phys. 93, 126 (2003).

[117] L. Thamizhmani, A. K. Azad, J. Dai, and W. Zhang, Appl. Phys. Lett. 86, 131111 (2005).

[118] A. Grüneis, G. Kresse, Y. Hinuma, and F. Oba, Phys. Rev. Lett. 112, 096401 (2014).

[119] R. H. French, J. Am. Ceram. Soc. 73, 477 (1990).

[120] M. Cardona and M. L. W. Thewalt, Rev. Mod. Phys. 77, 1173 (2005).

[121] G. Baldini, Phys. Rev. 128, 1562 (1962).

[122] R. Chrenko, Solid State Commun. 14, 511 (1974).

[123] G. Antonius, S. Poncé, E. Lantagne-Hurtubise, G. Auclair, X. Gonze, and M. Côté, Phys. Rev. B 92, 085137 (2015).

[124] A. Agui, S. Shin, and Y. Kumashiro, J. Phys. Soc. Jpn. 68, 166 (1999).

[125] C. D. Clark, P. J. Dean, and P. V. Harris, Proc. R. Soc. A 277, 312 (1964).

[126] R. Whited, C. J. Flaten, and W. Walker, Solid State Commun. 13, 1903 (1973).

[127] P. D. C. King, T. D. Veal, F. Fuchs, C. Y. Wang, D. J. Payne, A. Bourlange, H. Zhang, G. R. Bell, V. Cimalla, O. Ambacher, R. G. Egdell, F. Bechstedt, and C. F. McConville, Phys. Rev. B 79, 205211 (2009).

[128] G. Baldini and B. Bosacchi, Phys. Status Solidi B 38, 325 (1970).

[129] W. R. L. Lambrecht, C. Bhandari, and M. van Schilfgaarde, Phys. Rev. Mater. 1, 043802 (2017).

[130] M. Piacentini, D. W. Lynch, and C. G. Olson, Phys. Rev. B 13, 5530 (1976).

[131] J. P. Nery, P. B. Allen, G. Antonius, L. Reining, A. Miglio, and X. Gonze, Phys. Rev. B 97, 115145 (2018).

[132] T. Böker, R. Severin, A. Müller, C. Janowitz, R. Manzke, D. Voß, P. Krüger, A. Mazur, and J. Pollmann, Phys. Rev. B 64, 235305 (2001).

[133] D. M. Roessler and W. C. Walker, Phys. Rev. 166, 599 (1968).

[134] R. T. Poole, J. Liesegang, R. C. G. Leckey, and J. G. Jenkin, Phys. Rev. B 11, 5190 (1975).

[135] G. A. Sawatzky and J. W. Allen, Phys. Rev. Lett. 53, 2339 (1984).

[136] W. Bludau, A. Onton, and W. Heinke, J. Appl. Phys. 45, 1846 (1974).

[137] R. Humphreys, D. Bimberg, and W. Choyke, Solid State Commun. 39, 163 (1981). 
[138] B. Monserrat and R. J. Needs, Phys. Rev. B 89, 214304 (2014).

[139] H. Philipp, Solid State Commun. 4, 73 (1966).

[140] G. Kresse, M. Marsman, L. E. Hintzsche, and E. Flage-Larsen, Phys. Rev. B 85, 045205 (2012).

[141] K. Reimann and M. Steube, Solid State Commun. 105, 649 (1998).

[142] Y. Tezuka, S. Shin, T. Ishii, T. Ejima, S. Suzuki, and S. Sato, J. Phys. Soc. Jpn. 63, 347 (1994).

[143] B. Monserrat, Phys. Rev. B 93, 100301 (2016).

[144] A. Mang, K. Reimann, and S. Rübenacke, Solid State Commun. 94, 251 (1995).

[145] A. Mang, K. Reimann, S. Rübenacke, and M. Steube, Phys. Rev. B 53, 16283 (1996).

[146] F. Giustino, Rev. Mod. Phys. 89, 015003 (2017).

[147] M. J. van Setten, M. Giantomassi, X. Gonze, G.-M. Rignanese, and G. Hautier, Phys. Rev. B 96, 155207 (2017).

[148] C. Persson and A. F. da Silva, Appl. Phys. Lett. 86, 231912 (2005).

[149] L. Bernasconi, S. Tomić, M. Ferrero, M. Rérat, R. Orlando, R. Dovesi, and N. M. Harrison, Phys. Rev. B 83, 195325 (2011).

[150] R. Webster, L. Bernasconi, and N. M. Harrison, J. Chem. Phys. 142, 214705 (2015).

[151] Z.-h. Yang, F. Sottile, and C. A. Ullrich, Phys. Rev. B 92, 035202 (2015).

[152] F. Sottile, V. Olevano, and L. Reining, Phys. Rev. Lett. 91, 056402 (2003).

[153] L. Ley, R. A. Pollak, F. R. McFeely, S. P. Kowalczyk, and D. A. Shirley, Phys. Rev. B 9, 600 (1974).

[154] N. J. Shevchik, J. Tejeda, and M. Cardona, Phys. Rev. B 9, 2627 (1974).

[155] G. Martin, A. Botchkarev, A. Rockett, and H. Morkoç, Appl. Phys. Lett. 68, 2541 (1996).

[156] R. A. Powell, W. E. Spicer, and J. C. McMenamin, Phys. Rev. Lett. 27, 97 (1971).

[157] I. Jiménez, L. J. Terminello, D. G. J. Sutherland, J. A. Carlisle, E. L. Shirley, and F. J. Himpsel, Phys. Rev. B 56, 7215 (1997).

[158] A. L. Wachs, T. Miller, T. C. Hsieh, A. P. Shapiro, and T.-C. Chiang, Phys. Rev. B 32, 2326 (1985).
[159] F. J. Himpsel, L. J. Terminello, D. A. Lapiano-Smith, E. A. Eklund, and J. J. Barton, Phys. Rev. Lett. 68, 3611 (1992).

[160] L. Fiermans, R. Hoogewijs, G. de Meyer, and J. Vennik, Phys. Status Solidi A 59, 569 (1980).

[161] D. Waroquiers, A. Lherbier, A. Miglio, M. Stankovski, S. Poncé, M. J. T. Oliveira, M. Giantomassi, G.-M. Rignanese, and X. Gonze, Phys. Rev. B 87, 075121 (2013).

[162] P. Hao, Y. Fang, J. Sun, G. I. Csonka, P. H. T. Philipsen, and J. P. Perdew, Phys. Rev. B 85, 014111 (2012).

[163] M. Grimsditch, E. S. Zouboulis, and A. Polian, J. Appl. Phys. 76, 832 (1994).

[164] W. Wettling and J. Windscheif, Solid State Commun. 50, 33 (1984).

[165] M. H. Grimsditch and A. K. Ramdas, Phys. Rev. B 11, 3139 (1975).

[166] P. Son and R. Bartels, J. Phys. Chem. Solids 33, 819 (1972).

[167] R. I. Cottam and G. A. Saunders, J. Phys. C: Solid State Phys. 6, 2105 (1973).

[168] L. J. Bruner and R. W. Keyes, Phys. Rev. Lett. 7, 55 (1961).

[169] G. I. Csonka, J. P. Perdew, A. Ruzsinszky, P. H. T. Philipsen, S. Lebègue, J. Paier, O. A. Vydrov, and J. G. Ángyán, Phys. Rev. B 79, 155107 (2009).

[170] T. C. Chiang and F. J. Himpsel, Electronic structure of solids: Photoemission spectra and related data, in Landolt-Börnstein Group III Condensed Matter, Vol. 23A, edited by A. Goldmann and E.-E. Koch (Springer, Berlin, 1989).

[171] J. E. Ortega and F. J. Himpsel, Phys. Rev. B 47, 2130 (1993).

[172] R. A. Roberts and W. C. Walker, Phys. Rev. 161, 730 (1967).

[173] A. J. Garza and G. E. Scuseria, J. Phys. Chem. Lett. 7, 4165 (2016).

[174] L. Hedin, Phys. Rev. 139, A796 (1965).

[175] W. Kang and M. S. Hybertsen, Phys. Rev. B 82, 195108 (2010).

[176] A. Alkauskas and A. Pasquarello, Phys. B (Amsterdam) 401402, 670 (2007).

[177] F. Tran, Phys. Lett. A 376, 879 (2012).

[178] L. Lin, J. Chem. Theory Comput. 12, 2242 (2016).

[179] A. Damle, L. Lin, and L. Ying, J. Chem. Theory Comput. 11, 1463 (2015)

[180] I. Carnimeo, S. Baroni, and P. Giannozzi, arXiv:1801.09263. 